\title{
Multiferroics $\mathrm{BaCoX}_{2} \mathrm{O}_{7}(\mathrm{X}=\mathrm{P}$, As) compounds with incommensurate structural waves but collinear spins ingredients.
}

Bastien Leclercq ${ }^{\alpha}$, Angel M. Arévalo-López ${ }^{\alpha}$, Houria Kabbour ${ }^{\alpha}$, Sylvie DavieroMinaud ${ }^{\alpha}$, Alain Pautrat ${ }^{\beta}$, Tathamay Basu ${ }^{\beta}$, Claire V. Colin ${ }^{\gamma}$, Ranjana-Rani Das ${ }^{\gamma}$, Rénald David ${ }^{\alpha}$, Olivier Mentré ${ }^{\alpha, *}$

${ }^{\alpha}$ Univ. Lille, CNRS, Centrale Lille, Univ. Artois, UMR 8181 - UCCS - Unité de

Catalyse et Chimie du Solide, F-590oo Lille, France

$\beta$ Laboratoire CRISTMAT, UMR 6508-CNRS, ENSICAEN, 6 Bd. Du Maréchal Juin, 14000 Caen, France

r Univ. Grenoble Alpes, CNRS, Institut Néel, 38000 Grenoble, France

\begin{abstract}
A new paradigm in multiferroics has been observed in $\mathrm{BaCo}\left(\mathrm{X}_{2} \mathrm{O}_{7}\right)(\mathrm{X}=\mathrm{As}$, $\mathrm{P})$ compounds. They consist of $1 \mathrm{D}$-antiferromagnetic chains undulated by incommensurate structural modulations with unusually large atomic displacive waves, giving a mixed $1 \mathrm{D} / 2 \mathrm{D}$ "real" magnetic topology. The magnetic ground state is antiferromagnetic (AFM) with $\mathrm{k}=[1 / 20$ o leading to a non-modulated collinear spinlattice despite the aperiodic atomic framework, and allows developing spin-induced multiferroicity below $\mathrm{T}_{\mathrm{N}}$. Severe arguments against the identified mechanisms for type-II multiferroics, i.e. by inverse Dzyaloshinskii-Moriya (IDM), exchange striction and spin-dependent $p$ - $d$ hybridizations, suggest an original scenario in which the atomic waves, the collinear magnetic structure and magnetic dipole-dipole interactions may interact as crucial ingredients of the spin-induced ferroelectric phase. Here, the specific role of the $\mathrm{Co}^{2+}$ spin-orbit coupling (SOC) in the magnetoelectric (ME) phase diagram was demonstrated by comparison with the novel Heisenberg $\mathrm{BaFeP}_{2} \mathrm{O}_{7}$ isomorph, similarly structurally modulated. This compound shows a non-collinear modulated AFM ordering, while no ME coupling was detected in its case. Accordingly, both $\mathrm{BaCoX}_{2} \mathrm{O}_{7}$ and $\mathrm{BaFeP}_{2} \mathrm{O}_{7}$ undergo also metamagnetic transitions above 5-6 T promoted by the modulated distribution of spin-exchanges, but the spin-flop progressive alignment of the spins in the non-collinear spinstructure $\left(\mathrm{Fe}^{2+}\right.$ case) turns into an abrupt flip-like transition in the uniaxial spinstructure $\left(\mathrm{Co}^{2+}\right.$ case $)$.
\end{abstract}


Introduction: Low Dimensional magnetic materials may provide a platform for developing original multiferroic devices, due to the loss of symmetry elements compared to their $3 \mathrm{D}$-counterpart, often compatible with ferroelectricity ${ }^{[1,2]}$. From the perspective of fundamental physics, type II multiferroics, are very exciting because one of the orders (generally ferroelectricity) is a consequence of the other (generally antiferromagnetism). Besides their realization in Van der Waals layers or nano-devices, $1 \mathrm{D}$ or $2 \mathrm{D}$ subunits in bulk materials soften inter-block cohesion and spin interactions, which may reinforce spin-induced polarity in type-II multiferroics (MF), by exchange striction or competing mechanisms. A plethora of arguments for enhanced MF properties in such low-D systems are found in the literature such as i) their predominant insulating behavior necessary for dielectrics ${ }^{[3,4]}$, ii) the persistence of spin ordering in low-D units at relatively high temperature counterpart ${ }^{[5]}$, iii) their good compatibility with the main mechanisms for ferroelectricity of spin origin ${ }^{[6,7]}$, iv) the frustration in low-D topologies playing for spin driven polar atomic shifts ${ }^{[8]}$, v) the possibility of metamagnetic transitions aligning isolated magnetic units with enhanced magneto-electric (ME) exchanges at magnetization steps, see $\mathrm{CoV}_{2} \mathrm{O}_{6}{ }^{[9,10]}$. In addition, the effects of magnetic fields on low-D structures open the route to other fascinating phenomena, such as Bose-Einstein condensation or novel field-induced magnetic states such as skyrmions ${ }^{[1]}$. Here highly anisotropic $\mathrm{Co}^{2+}$ spin-chains may generate field-induced particularities, like incommensurate spin density waves (SDW) in the screw AFM chain of $\mathrm{BaCO}_{2} \mathrm{~V}_{2} \mathrm{O}_{8}{ }^{[12]}$, metamagnetic spin-flip versus spinflop in $\mathrm{Co}^{2+}$ chain-systems ${ }^{[13]}$, Spin-dynamics and low-field spin alignments in canted-1D inorganic systems; i.e. $\mathrm{BaCo}_{2}\left(\mathrm{As}_{3} \mathrm{O}_{6}\right)_{2} \cdot\left(\mathrm{H}_{2} \mathrm{O}\right)_{2}{ }^{[14]}$ analogue to hybrid singlechain-magnets (SCM's) ${ }^{[15,16]}$. Although ignored up to day, the access to non-collinear magnetic structures and magnetization steps suggest attractive ME properties.

Chemically speaking, most of the low-D cited phases were isolated in $\mathrm{BaO}$ CoO- $\mathrm{X}_{2} \mathrm{O}_{5}$ systems $(\mathrm{X}=\mathrm{P}, \mathrm{V}, \mathrm{As})$ which highlights the promoting role of large $\mathrm{Ba}^{2+}$ cations and $\mathrm{XO}_{4}$ groups such as phosphates as spacers between low-D subunits ${ }^{[17,18]}$. The transition metal ion itself dictates the magnetic behaviours through spin orbit coupling (SOC) making the $\mathrm{Fe}^{2+}, \mathrm{Co}^{2+}$ tandem relevant for contrasted behaviours, see the Ising 2D-ferromagnetic $\mathrm{BaFe}\left(\mathrm{PO}_{4}\right)_{2}$ versus quasi-2 $\mathrm{D} \mathrm{XY} \mathrm{Ba}\left(\mathrm{CoAsO}_{4}\right)_{2}{ }^{\left[{ }^{19,20}\right]}$. Amongst the variety of reported compounds, $\mathrm{BaCoX}_{2} \mathrm{O}_{7}(\mathrm{X}=\mathrm{P}, \mathrm{As})$ appear as good candidates for developing spin-induced multiferroicity ${ }^{[21]}$ combining low-D topologies and aperiodic magnetic periods ${ }^{[22,23]}$. Although the crystal structures reveal spatially isolated AFM-1D chains of ferromagnetic dimers, the existence of metamagnetic $\mathrm{M}(\mathrm{H})$ steps around $\mathrm{H}=5-6 \mathrm{~T}$ questions about the real magnetic topology. In reality, incommensurate structural modulations with unusually strong atomic displacive waves, create undulated $1 \mathrm{D}$-chains which form all together singular quasi $2 \mathrm{D}$ layers. Here we propose that the atomic displacive waves assorted with a specific magnetic structure and magnetic dipole-dipole couplings interact as crucial ingredients for an original multiferroic scenario, verified experimentally. In this aim, we have prepared the original $\mathrm{BaFe}\left(\mathrm{P}_{2} \mathrm{O}_{7}\right)$ isomorph compound for rationalization based on the labile degree of SOC, within an extended series. 
$\mathrm{BaFeP}_{2} \mathrm{O}_{7}$ Structural modulation and analogy with the $\mathrm{Co}^{2+}$ phases: Polycrystalline samples with single or mixed $\mathrm{Fe}^{2+} / \mathrm{Co}^{2+}$ cations prepared in the frame of this work are shown in Figure 1a and important notes about the solid state chemistry, synthetic routes and their preliminary physico-chemical characterizations are given in the supplementary, So.

Dealing with the rationalization of magnetic and ME properties of the $\mathrm{BaMX}_{2} \mathrm{O}_{7}$ series, a relevant aspect developed during our work concerns complex spin-lattice coupling within incommensurately modulated materials. The crystal structure of the original $\mathrm{BaFeP}_{2} \mathrm{O}_{7}$ member is modulated similarly to $\mathrm{BaCoX}_{2} \mathrm{O}_{7}(\mathrm{X}=\mathrm{P}, \mathrm{As}, \mathrm{V})^{[21,24]}$. Its triclinic (S.G. P-1, cell parameters $a=5.3855(2) \AA, b=7.5783(3) \AA, c=7.1496(4) \AA, \alpha=$ $\left.102.302(3)^{\circ}, \beta=93.811(3)^{\circ}, \gamma=91.315(3)^{\circ}\right)$ average structure, consists of infinite chains of edge sharing $\mathrm{Fe}_{2} \mathrm{O}_{8}$ square-pyramid dimers running along the $a$-axis (see Figure $1 b)$, connected by pyro-arsenates $\mathrm{As}_{2} \mathrm{O}_{7}$ bridges but disconnected in the ac plane. These layers are stacked along the $b$-axis and separated by $\mathrm{Ba}^{2+}$ cations. Single crystal data show satellites spots fully indexed using the $q=[0.16249(3), 0.06573(5)$, $0.45116(4)]$ incommensurate modulation vector similarly to the Co and Ni variants $[21,24-26]$.

Its $3 \mathrm{D}+1$ crystal structure was solved in the $\mathrm{P}-1(\alpha, \beta, \gamma)_{0}$ superspace group, after integration of satellites with order up to $\mathrm{m}=3$. Finally, refining $3^{\text {rd }}$ order displacive waves for all $\mathrm{Ba}, \mathrm{Fe}, \mathrm{P}$ cations and $\mathrm{O}_{3}$ anion and $1^{\text {st }}$ order waves for other oxygen atoms with $\mathrm{R}_{(\mathrm{I}>3 \sigma(\mathrm{I}))}=5.48 \%$, see Supplementary Tables 1 for details of the structural refinement (characteristics, positions and thermal displacement). In the same way as the $\mathrm{Co}^{2+}$ compounds ${ }^{21,24}$ the main atomic displacement occurs along the $c$ direction, i.e. between individual chains in the ac plane, such that the modulated $\mathrm{Co}-\mathrm{O}_{7}$ bond distance varies between $\sim 2.2$ and $\sim 3.7 \AA$, locally connecting or not the $1 \mathrm{D}$ - chains into $2 \mathrm{D}$-planes ${ }^{[21]}$. To the best of our knowledge, such "giant" cation-anion modulated separation is uncommon, much above those observed in $(\mathrm{LaS})_{1.196} \mathrm{VS}_{2}$ despite important V-V displacive waves in the latter $(\sim 3.05-3 \cdot 71 \AA)^{[27]}$.

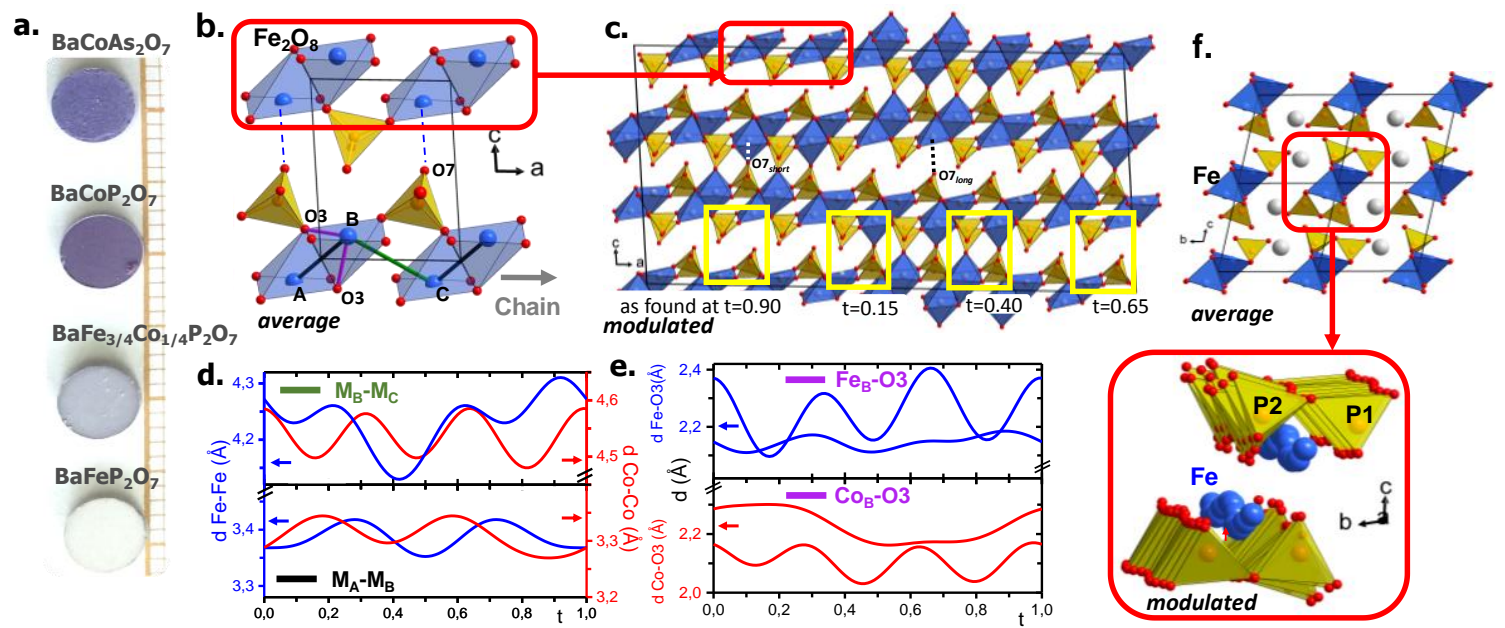

Figure 1 BaMX $\mathrm{B}_{2} \mathrm{O}_{7}$ Crystal structure: a) Various $\mathrm{BaMX}_{2} \mathrm{O}_{7}(\mathrm{M}=\mathrm{Co}, \mathrm{Fe}, \mathrm{X}=\mathrm{P}$, As) samples and colour changes. $\boldsymbol{b})$ average unit cell projected along $b$ with with color code for relevant distances. c) $7 \times 1 \times 3$ supercell, modulated unit cell with highlight of local structures with Co-Co distances which correspond to the 
average cells at various t as defined in the text. d-e) evolution of $M_{B}-M_{C}, M_{A}-M_{B}, F_{B}-O_{3}, \mathrm{Co}_{B}-O_{3}$ distances vs. t.f) average and modulated chains and next $\mathrm{As}_{2} \mathrm{O}_{7}$ groups along the a-direction.

Similarly to $\mathrm{BaCoX}_{2} \mathrm{O}_{7}^{[21]}$, in $\mathrm{BaFeP}_{2} \mathrm{O}_{7}$ the ${ }_{3} \mathrm{D}+1$ symmetry strongly affects the connectivity scheme. Between two next layers along $c$, it is mainly anti-phased with those of the next interleave, creating a distribution of compact versus dispersed areas, depending on the strongly modulated $\mathrm{Fe}--\mathrm{O}_{7}$ bond distances ( $\sim 2.3$ to $3.7 \AA$, see $\mathrm{S} 1$ ) within the $\mathrm{FeO}_{5+1}$ polyhedra, see Figure 1c. Magnetically, it brings a rare mixed $1 \mathrm{D}-2 \mathrm{D}$ topology. Along the chains axis ( $a$-axis), we found that the modulated M-M separation (inside and between the dimers) keep the same amplitude for $\mathrm{BaCoX}_{2} \mathrm{O}_{7}$ and $\mathrm{BaFeP}_{2} \mathrm{O}_{7}$ and can be considered as weak (3.35 to $3.42 \AA$ in the dimers, 4.13 to $4.32 \AA$ between them, see $\left.\mathrm{S}_{1}\right)$ compared to the undulations along the $c$-axis $\left(\mathrm{d}_{\mathrm{Fe}-\mathrm{Fe}} \sim 5.4\right.$ to $6.3 \AA$ ), as plotted along the real space $t$-axis (where $\mathrm{t}=\mathrm{x} 4-\vec{q} \cdot \vec{r}, \vec{q}$ is the modulation vector and $\vec{r}$ an average position in physical space) in Figure $1 \mathrm{~d}$. On the opposite, the modulated $\mathrm{FeO}_{5+1}$ distortion is amplified compared to $\mathrm{CoO}_{5+1}$ especially dealing with the $\mathrm{M}-\mathrm{O}_{3}$ bonds involved in the shared edge dimeric connection, see Figure 1e. All together, we are dealing with undulated chains of dimers that mainly preserve the Fe-Fe distances "in" and "between" the dimers due to in-phase Fe waves but assorted with strong local distortion of the polyhedra due to the modulated bending of the $\mathrm{P}_{2} \mathrm{O}_{7}$ groups, see Figure if.

\section{Magnetic Properties against cationic specificities : Despite similar} incommensurate atomic displacement waves, the magnetic properties of $\mathrm{BaFeP}_{2} \mathrm{O}_{7}$ differ significantly from those of $\mathrm{BaCoX}_{2} \mathrm{O}_{7}(\mathrm{X}=\mathrm{P}, \mathrm{As})$. The main characteristics of the three key compounds are listed in Table 1. For the three cases, the fundamental state is antiferromagnetic but the magnetic structure and field-dependence differ by contrasted degrees of SOC whose influences on the ME properties are of primary importance. The contrasted $\theta_{\mathrm{CW}}$ values between the $\mathrm{Co}^{2+}$ arsenate $\left(\theta_{\mathrm{CW}}=-3.75 \mathrm{~K}\right)$ and phosphate $\left(\theta_{\mathrm{CW}}=-21.7 \mathrm{~K}\right)$ are in good agreement with the hierarchical exchange values latter discussed and the aptitude of $\mathrm{PO}_{4^{3-}}$ oxo-anions for strong magnetic transmission compared to $\mathrm{AsO}_{4}{ }^{3-}$ ones ${ }^{[28,29]}$.

$\mathrm{BaCoX}_{2} \mathrm{O}_{7}(\mathrm{X}=$ As and $\mathrm{P})$ shows magnetization plateaus when increasing the applied field assigned to $\mathrm{Ms} / 2^{[24]}$ or $\mathrm{Ms} / 3 \mathrm{M}(\mathrm{H})$ steps ${ }^{[2]}$ while the saturation is not fully reached at $14 \mathrm{~T}$. In both compounds the rise of the plateaus is abrupt, reminiscent of spin-flip like transitions well supported by Ising $\mathrm{Co}^{2+}$ single ions due to the strong SOC, see the $\mu_{\text {eff }}$ values in Table 1 . In fact, our single crystal measurements on $\mathrm{BaCoAs}_{2} \mathrm{O}_{7}$ confirm a broad metamagnetic-like component above $5.5 \mathrm{~T}$ especially pronounced along the [100] easy magnetic axis, while a component along the [0o1] is growing at higher field (ca. 9T), see Figure 2a. The sum along the three crystallographic axes creates pseudo-inflexions coincidentally assigned to specific steps. Such pseudo $\mathrm{M}=\mathrm{M}_{\mathrm{S}} / 3$ magnetic steps were already reported in $(\mathrm{CuBr}) \mathrm{Sr}_{2} \mathrm{Nb}_{3} \mathrm{O}_{10}{ }^{[30,31]}$.

$\mathrm{BaFeP}_{2} \mathrm{O}_{7}$ is paramagnetic down to $c a$. $20 \mathrm{~K}$ and also shows a broad $\chi(\mathrm{T})$ maximum at $16.8 \mathrm{~K}$ reminiscent of low-D magnetism similar to the $\mathrm{Co}^{2+}$ compounds, see Figure $2 \mathrm{~b}$. Neel ordering occurs at $10.3 \mathrm{~K}$ as confirmed by the heat capacity $\lambda$-type anomaly (see Figure $2 \mathrm{c}, \mathrm{d}$ and Supplementary Figure 2 for extended temperature 
range). In contrast, the $\mathrm{M}(\mathrm{H})$ magnetization plots differ significantly between $\mathrm{Co}^{2+}$ and $\mathrm{Fe}^{2+}$ compounds as shown Figure $2 \mathrm{~b}$. In the later, $\mathrm{M}(\mathrm{H})$ increases progressively even at low field, followed by a smooth metamagnetic transition at $6 \mathrm{~T}$ with a rapid increase of the magnetization and weak hysteretic effects between the field increasing and decreasing branches. We observed similar behaviours for $\mathrm{Fe} / \mathrm{Co}$ solid solution, see Supplementary Figure 3 for the plots and a comparison between pertinent physical parameters. Comparatively to the almost reached saturation in pure $\mathrm{Co}^{2+}$ phases, $\mathrm{BaFeP}_{2} \mathrm{O}_{7} \mathrm{M}(\mathrm{H})$ barely reaches $3 / 4^{\text {th }}$ of $\mathrm{Ms}$ (estimated to $4 \mu_{\mathrm{B}}$ for $\mathrm{S}=2 \mathrm{Fe}^{2+}$ ) at $14 \mathrm{~T}$ which higlight versatile magnetocrystalline anisotropy. Alignment of the powder at $330 \mathrm{~K} / 9 \mathrm{~T}$ in a gel did not modify the $\mathrm{M}(\mathrm{H})$ plot as expected in absence of an easy magnetic axis, see Figure $2 \mathrm{~b}$, contrarily to $\mathrm{Co}^{2+}$ based series of compounds ${ }^{[24]}$. This spin flop-like transition suggests Heisenberg isotropic $\mathrm{Fe}^{2+}$ individual ions in

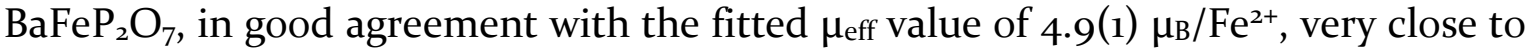
the spin-only moments for $\mathrm{S}=2 \mathrm{Fe}^{2+}$ ions $\left(4.89 \mu_{\mathrm{B}}\right)$ which refute significant SOC contribution, see Table 1 , in contrast with the common behavior of $\mathrm{Fe}^{2+}$ based oxides [20]. The 3-D Heisenberg character of $\mathrm{BaFe}_{2} \mathrm{P}_{2} \mathrm{O}_{7}$ is comforted by the $\beta$ critical exponent further refined to 0.36(5) from PND data, in excellent agreement to the 0.367 expected value, Supplementary Figure 4. The situation of $\mathrm{BaFeP}_{2} \mathrm{O}_{7}$ versus $\mathrm{BaCoX}_{2} \mathrm{O}_{7}$ is reminiscent of the recent report of the spin-flop occurring in $\mathrm{BaCO}_{2} \mathrm{As}_{2} \mathrm{O}_{8.2} \mathrm{H}_{2} \mathrm{O}$ within a zigzag spin structure versus the spin-flip transition in $\mathrm{Co}_{2} \mathrm{As}_{2} \mathrm{O}_{7} \cdot 2 \mathrm{H}_{2} \mathrm{O}$ with a collinear spin ordering ${ }^{[13]}$. On this basis, the "flip" versus "flop" like transition types for $\mathrm{Co}^{2+}$ and $\mathrm{Fe}^{2+}$ respectively suggest uniaxial-collinear spin ordering in $\mathrm{BaCoX}_{2} \mathrm{O}_{7}$ (experimentally verified in ${ }^{[21]}$ ) against Heisenberg spins with a plausible non-colinear spin-structure in $\mathrm{BaFeP}_{2} \mathrm{O}_{7}$. Here the softening of a macroscopic easy axis may result from the modulated $\mathrm{Fe}^{2+}$ atomic strings and locally modulated $\mathrm{FeO}_{5+1}$ distortions along the chains.

The specific heats of $\mathrm{BaCoAs}_{2} \mathrm{O}_{7}$ and $\mathrm{BaFeP}_{2} \mathrm{O}_{7}$ confirm in the former the preservation of the $\lambda$-peak at $9 \mathrm{~T}$ and highlights a robust/uniaxial AFM state (see Figure $2 \mathrm{c}, \mathrm{d}$ ). Contrarily, in the latter the vanishing of the $\lambda$-type into a broad kink at $\mathrm{T}_{\mathrm{N}}$ upon increasing the field validates a "forced spin-aligned" state similar to a paramagnet under high field. It resembles the high-field state of $\mathrm{BaCo}_{2} \mathrm{~V}_{2} \mathrm{O}_{8}$ in which the field induces an order-disorder transition ${ }^{[12,32,33]}$. 

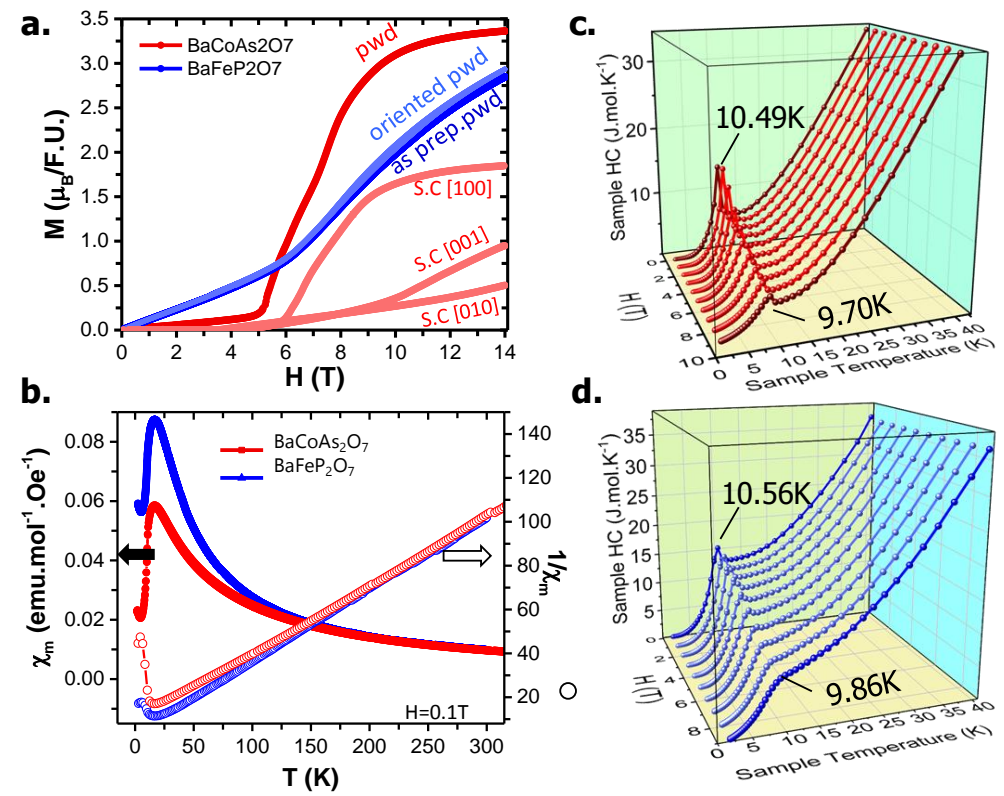

Figure 2 Magnetic Characterizations a) $\mathrm{M}(\mathrm{H})$ plot (2K) for $\mathrm{BaCoAs}_{2} \mathrm{O}_{7}$ (red) and $\mathrm{BaFeP}_{2} \mathrm{O}_{7}$ (blue) powder and single crystal samples (S.C). In the former, the main contribution falls along the a axis, i.e. the chains direction. In the latter, attempts to orientate the powder using eicosane gel at $9 \mathrm{~T}$ show the absence of easy magnetic axis. b) $\chi_{\mathrm{m}}(\mathrm{T})$ and $1 / \chi_{\mathrm{m}}(\mathrm{T})$ plots between 300 and $2 \mathrm{~K}$ at $0.1 \mathrm{~T}$. c) Specific heat measurement from o to $9 \mathrm{~T}$ and 40 to $2 \mathrm{~K}$ for $\mathrm{BaCoAs}_{2} \mathrm{O}_{7}$, showing the preservation of the $\mathrm{T}_{\mathrm{N}}$-peak. d) Same data for $\mathrm{BaFeP}_{2} \mathrm{O}_{7}$, with vanishing of the $\lambda$-peak anomaly a $\mathrm{T}_{\mathrm{N}}$ into a schottky-like anomaly under field.

Table 1 1 Main structural and magnetic features for $\mathrm{BaCoAs}_{2} \mathrm{O}_{7}, \mathrm{BaCoP}_{2} \mathrm{O}_{7}$ and $\mathrm{BaFeP}_{2} \mathrm{O}_{7}$ compounds (left to right).

\begin{tabular}{|c|c|c|c|}
\hline compound & $\mathrm{BaCoAs}_{2} \mathrm{O}_{7}$ & $\mathrm{BaCoP}_{2} \mathrm{O}_{7}$ & $\mathrm{BaFeP}_{2} \mathrm{O}_{7}$ \\
\hline & \multicolumn{3}{|c|}{ Crystallograpy, superspace group $\mathrm{P}-1(\alpha, \beta, \mathrm{Y}) 0$} \\
\hline$a(\AA), \alpha\left({ }^{\circ}\right)$ & $5.5386(2), 101.5001(2)$ & $5.3230(6), 101.217(4)$ & $5.3855(2), 102.302(3)$ \\
\hline$b(\hat{A}), \beta\left({ }^{\circ}\right)$ & $7.7581(3), 96.4724(2)$ & 7.5799(4), 95.809(8) & $7.5783(3), 93.811(3)$ \\
\hline$c(\ddot{A}), y\left({ }^{\circ}\right)$ & 7.2947(3), $91.6781(2)$ & 7.1174(3), 90.681(8) & 7.1496(4), 91.315(3) \\
\hline \multirow{4}{*}{$\begin{array}{c}\text { Volume }\left(\AA^{3}\right) \\
\text { q vector }\end{array}$} & $304.76(2)$ & $280.09(2)$ & $284.26(2)$ \\
\hline & $0.13571(4)$ & $0.146(4)$ & $0.1625(1)$ \\
\hline & $0.0781(1)$ & $0.075(2)$ & $0.0657(1)$ \\
\hline & $0.4768(1)$ & $0.470(2)$ & $0.4512(1)$ \\
\hline & \multicolumn{3}{|c|}{ Magnetic Properties } \\
\hline $\mathrm{T}_{\mathrm{N}}(\mathrm{K} /)$ & 10.68 & 10.41 & 10.28 \\
\hline$\mu_{\text {eff }}\left(\mu_{B} / F . U.\right) /($ calc. $)$ & $4.35 / 3.87$ & $5.1 / 3.87$ & $4.9 / 4.89$ \\
\hline $\boldsymbol{\theta}_{\mathrm{cw}}(\mathrm{K})$ & -3.75 & -21.7 & -10.88 \\
\hline & \multicolumn{3}{|c|}{ DFT Calculs (GGA+U average cell) $\mathrm{U}=4 \mathrm{eV} \mathrm{Co}{ }^{2+} / 6 \mathrm{eV} \mathrm{Fe}{ }^{2+}$} \\
\hline $\begin{array}{c}J_{\text {dimer }}(K / m e V) \\
d_{M-M}(\AA)\end{array}$ & $\begin{array}{c}2.7 / 2.310^{-2}(\mathrm{FM}) \\
4.53\end{array}$ & $\begin{array}{c}+23.1 / 0.2(\mathrm{FM}) \\
3.25\end{array}$ & $\begin{array}{c}+1.04 / 8.910-3(\mathrm{FM}) \\
3.38\end{array}$ \\
\hline $\begin{array}{c}\mathrm{J}_{\text {chain }}(\mathrm{K} / \mathrm{meV}) / \\
\mathbf{d}_{\mathrm{M}-\mathrm{M}}(\AA)\end{array}$ & $\begin{array}{c}-7.5 /-6.410^{-2}(\mathrm{AFM}) / \\
5.54\end{array}$ & $\begin{array}{c}-13.1 /-0.11(\mathrm{AFM}) / \\
5.32\end{array}$ & $\begin{array}{c}-2.8-/ 2.410^{-2}(\mathrm{AFM}) / \\
5.38\end{array}$ \\
\hline $\mathrm{J}_{\mathrm{c}}(\mathrm{K})$ & $-0.8 / 5.98-6.02$ & not calc. / $5.86(2 x)$ & not calc. / 5.79-5.90 \\
\hline \multirow[t]{2}{*}{$\mathbf{J}_{\text {dimer }} / \mathbf{J}_{\text {chain }}$} & -0.36 & -1.76 & -0.37 \\
\hline & \multicolumn{3}{|c|}{ Ground State AFM spin Structure (2 K) } \\
\hline Spins & Collinear non Modul.. & Collinear non Modul.. & Tilted Modul. \\
\hline
\end{tabular}




\begin{tabular}{|c|c|c|c|}
\hline Ref. Moment $\left(\mu_{B}\right)$ & $3.452(58)$ total & 3.65(12) total & 3.3(1) average \\
\hline $\mathbf{R}_{\text {magn. }} \%$ & 6.76 & 2.09 & 1.05 \\
\hline Instrument & D2B (ILL), $\lambda=2.4 \AA$ & WISH (ISIS), T.O.F & D1B (ILL), $\lambda=2.52 \AA$ \\
\hline & \multicolumn{3}{|c|}{ Metamagnetism } \\
\hline$H_{c}(T)$ (inflexion) & 5.14 & 4.96 & 6.13 \\
\hline$M_{14 T}(T)$ & 3.3 & 3.4 & 2.9 \\
\hline Allure & Sharp & Sharp & Smooth \\
\hline \multirow[t]{2}{*}{ Easy magn.axis } & [100] & [100] & None \\
\hline & \multicolumn{3}{|c|}{ Magnetic dipole-dipole Interaction } \\
\hline $\mathbf{J d d}_{\text {dimer }}(\mathrm{meV})$ & $+7.15 \times 10^{-3}$, repuls. & $+6.00 \times 10^{-3}$, repuls. & $-1.01 \times 10^{-2}$, attract. \\
\hline$J_{d d}$ chain $(m e V)$ & $+1.88 \times 10^{-3}$, repuls & $+7.21 \times 10^{-3}$, repuls. & $-1.87 \times 10^{-3}$, attract. \\
\hline $\mathrm{Jdd}_{\text {dimer }}$ total (meV) & $+7.32 \times 10^{-3}$, repuls. & $+6.71 \times 10^{-3}$, repuls. & $-8.96 \times 10^{-3}$, attract. \\
\hline$J_{d d}$ chain total (meV) & $+2.36 \times 10^{-3}$, repuls & $+6.36 \times 10^{-3}$, repuls. & $-1.99 \times 10^{-3}$, attract. \\
\hline $\mathbf{J d d}_{\text {dimer }} / \mathbf{J d d}_{\text {chain }}$ & 3.098 & 1.055 & 4.504 \\
\hline
\end{tabular}

AFM ground states vs. modulation: The contrasted magnetic responses of the $\mathrm{Co}^{2+}$ vs. $\mathrm{Fe}^{2+}$ compounds in terms of metamagnetism and degrees of SOC picture different fundamental AFM states at zero field. Indeed, as previously reported, the $\mathrm{BaCo}_{2} \mathrm{P}_{2} \mathrm{O}_{7}$ AFM magnetic ground state is described by a single $\mathrm{k}=(1 / 2 \mathrm{O} 0)$ propagation vector with respect to a collinear spin structure, Ising compatible ${ }^{[21]}$. In contrast the isotropic $\mathrm{Fe}^{2+}$ single ions in $\mathrm{BaFeP}_{2} \mathrm{O}_{7}$ suggests important spin-lattice coupling and maybe influenced by the structural modulation, as confirmed below. The main phenomena evidenced by PND along temperature and applied fields are shown in Figure 3.

The magnetic structure of $\mathrm{BaCoP}_{2} \mathrm{O}_{7}$ was previously refined ignoring the structural modulation, leading to $\mathrm{M}_{\mathrm{Co}}=3.73 \mathrm{O}(1) \mu_{\mathrm{B}}$ nearly along the a-axis, i.e the chain direction $^{[21]}$. Dealing with group theory, symmetry mode analysis using Isodistort ${ }^{[34,35]}$ shows that coupling the nuclear incommensurate structural vector with the magnetic $\mathrm{X}$ point $(1 / 2,0,0)$ results in two possible magnetic irreducible representations $\mathrm{GP}_{1} \mid \mathrm{mX}_{1}+$ and $\mathrm{GP}_{1} \mid \mathrm{mX}_{1}$. The difference between them is an origin shift of the magnetic inversion ( $\left.1^{\prime}\right)$ by $1 / 4$ along the $a$ direction. Practically it yields FM dimers AFM coupled ( $\left.\mathrm{GP}_{1} \mid \mathrm{mX}_{1}+\right)$ against AFM dimers FM coupled (GP1 $\left.\mid \mathrm{mX}_{1}-\right)$. The refined model clearly validates the former model, in good agreement with the DFT calculated spin exchanges detailed below.

For $\mathrm{BaCoP}_{2} \mathrm{O}_{7}$ (ILL-France, D2b data), NPD refinements converge to $\mathrm{M}_{\mathrm{Co}_{0}}=3.65(12) \mu_{\mathrm{B}}$ similar to the previous report (see Table 1, Figure 4a,d and Supplementary Figure 4). The moments are nearly confined in the $(a b)$ planes with the greatest component along $a$, i.e. the chain axis. Attempt to affect the magnetic structure using the structural satellites lead to modulated amplitudes smaller than $2 \sigma$, see Table 3 , leading to a non-modulated picture with AFM chains of collinear and equal FM spindimers, along $a$, a non-trivial distribution taking into account the strongly undulated chains. 
For $\mathrm{BaCoAs}_{2} \mathrm{O}_{7}$ (ISIS spallation source-UK, WHISH data), the same approach at o T results into similar unmodulated spin moment values $\left(M=3.45(6) \mu_{B}\right)$ see Table 3 , but one should note a greater $\mathrm{M}_{\mathrm{z}}$ component, i.e. toward the interchain region (see, Figure $4 \mathrm{~b}, \mathrm{~d}$ and also Supplementary Figure 4 for the extended range diagram and details of the refinement). In summary at o T for both $\mathrm{BaCoX}_{2} \mathrm{O}_{7}$ materials $(\mathrm{X}=\mathrm{P}$, As), FM dimers couple antiferromagnetically mainly along the structural chains, parallel to the $a$ direction, see Figure $4 \mathrm{a}, \mathrm{b}$. The spins ignore the structural modulation and lie almost confined into the equatorial plane of the $\mathrm{CoO}_{6}$ octahedra, this reflects the strong Ising-like ion anisotropy of $\mathrm{Co}^{2+}$ sensitive to the local orbital overlapping.

For $\mathrm{BaFeP}_{2} \mathrm{O}_{7}$ (ILL, France, Dib data ${ }^{[36]}$ ) the situation is quite different, where in addition to the temperature independent structural satellites ( $\mathrm{q}$ vector), both $\mathrm{k}_{1}=$ $(1 / 20$ o $)$ AFM satellites and their $1^{\text {st }}$ order satellites modulated by $\mathrm{k}_{2}=\mathrm{q}$ (the structural modulation mode) grow below $\mathrm{T}_{\mathrm{N}}$. The $(1 / 2,0,1)-\mathrm{k}_{2},(1 / 2,-1,0)-\mathrm{k}_{2}$ and $(1 / 2,1,0)+\mathrm{k}_{2}$ reflexions show the strongest intensity, see Figure $3 \mathrm{~b}$. The average AFM state is similar than for the $\mathrm{Co}^{2+}$ materials giving FM dimers AFM coupled along the chains but confined in the $(a, c)$ plane this time, i.e. almost normal to the spins in the two $\mathrm{Co}^{2+}$ compounds, a direction much more sensitive to the chain undulation. Indeed, the effect of the modulation of the AFM structure with respect to a two k-magnetic structure gives a significant wave component along c, leaving mainly FM dimers with modulated amplitude and directions. The average moment is 3.31(1) $\mu_{B}$ with local values between 2.5-4.2 $\mu_{\mathrm{B}}$, see Figure 4c,d. The spin lattice coupling dictates the spin orientation influenced by the local changes and is responsible for the smooth spin alignment increasing the field, contrarily to the uniaxial $\mathrm{Co}^{2+}$ compounds with an abrupt $\mathrm{M}(\mathrm{H})$ step. Fitting the $\mathrm{M} / \mathrm{M}_{\max }(\mathrm{T})$ evolution using $\mathrm{A}\left(1-\left(\mathrm{T} / \mathrm{T}_{\mathrm{N}}\right)\right)^{\beta}$ yields $\beta=0.36(9)$, as expected for 3-D Heisenberg systems (0.367), see Supplementary Figure 4 (Rietveld refinement plot, non modulated structure representation, and temperature evolution of the refined magnetic moments). The refined moment and wave components of the modulation are listed in Table 2 , for the three compounds. 

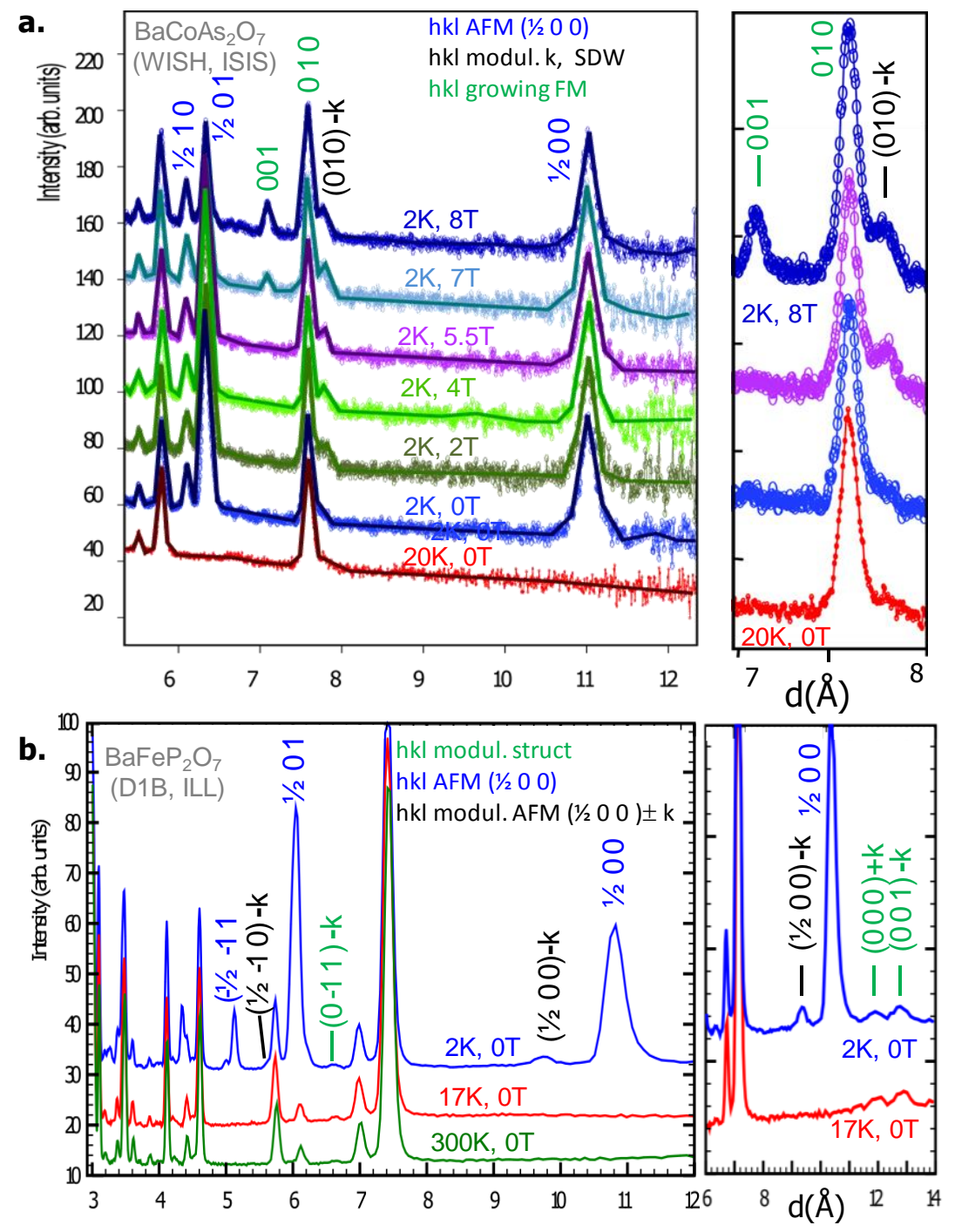

Figure 3 | Powder Neutron Diffraction: a) PND patterns for $\mathrm{BaCoAs}_{2} \mathrm{O}_{7}$ of the (WISH, ISIS) from $20 \mathrm{~K}, \mathrm{o} T$ to $2 \mathrm{~K}, 8 \mathrm{~T}$ (bottom up). It shows the $\mathrm{k}_{\mathrm{AFM}}=\left(1 / 2 \mathrm{o}\right.$ o) satlellites below $\mathrm{T}_{\mathrm{N}}$, the appearing of the incommensurate $(\mathrm{ksDW}=\mathrm{q})$ SDW magnetic satellites above $2 \mathrm{~T}$ and the growing of FM fundamental Bragg components above $7 \mathrm{~T}$. b) PND patterns for $\mathrm{BaFeP}_{2} \mathrm{O}_{7}$ (Dib, ILL) versus temperature with highlights of the incommensurate structural (q) peaks in green, the AFM k1 $=(1 / 20$ o $)$ satellites in blue and their $2 \mathrm{k}$ modulated satellites by $(\mathrm{k} 2=\mathrm{q})$ in black. On the right, enlargement of pertinent areas.

Table $3 \mid$ Magnetic Modulation refined moments and wave components for $\mathrm{BaMX}_{2} \mathrm{O}_{7}$ compounds using the mixed refinements of the modulated crystal structure and of the modulated AFM spin structure. Only the sinusoidal term along $x$ for $\mathrm{BaFeP}_{2} \mathrm{O}_{7}(>3 \sigma)$ is significant, as confirmed by the observation of two-k satellites.

\begin{tabular}{|c|c|c|c|c|c|c|}
\hline & Atom & Wave & Along a & Along b & Along c & $\mathbf{M}\left(\mu_{\mathrm{B}}\right)$ \\
\hline $\mathrm{BaFeP}_{2} \mathrm{O}_{7}$ & $\mathrm{Fe}$ & $\begin{array}{c}0 \\
\operatorname{Sin} 1 \\
\operatorname{Cos} 1 \\
\end{array}$ & $\begin{array}{c}2.69(5) \\
0.9(2) \\
0.3(3) \\
\end{array}$ & $\begin{array}{c}-0.28(9) \\
-0.6(3) \\
0 \\
\end{array}$ & $\begin{array}{c}2.02(6) \\
0 \\
-0.4(2) \\
\end{array}$ & $\begin{array}{c}3.31(12) \\
1.1(4) \\
0.5(3)\end{array}$ \\
\hline $\mathrm{BaCoP}_{2} \mathrm{O}_{7}$ & Co & $\begin{array}{c}0 \\
\operatorname{Sin} 1 \\
\text { Cos1 } \\
\end{array}$ & $\begin{array}{c}2.92(4) \\
0.2(3) \\
-0.2(3) \\
\end{array}$ & $\begin{array}{c}2.10(4) \\
-0.5(3) \\
-0.4(2) \\
\end{array}$ & $\begin{array}{c}0.30(7) \\
0.1(4) \\
-0.1(4) \\
\end{array}$ & $\begin{array}{c}3.8(1) \\
0 \\
0 \\
\end{array}$ \\
\hline $\mathrm{BaCoAs}_{2} \mathrm{O}_{7}$ & Co & $\begin{array}{c}0 \\
\operatorname{Sin} 1 \\
\operatorname{Cos} 1 \\
\end{array}$ & $\begin{array}{l}3.06(2) \\
0.3(2) \\
0.1(2) \\
\end{array}$ & $\begin{array}{l}1.79(2) \\
0.0(3) \\
0.0(3) \\
\end{array}$ & $\begin{array}{c}1.02(4) \\
-0.3(2) \\
0.2(2) \\
\end{array}$ & $\begin{array}{c}3.44(5) \\
0 \\
0\end{array}$ \\
\hline
\end{tabular}



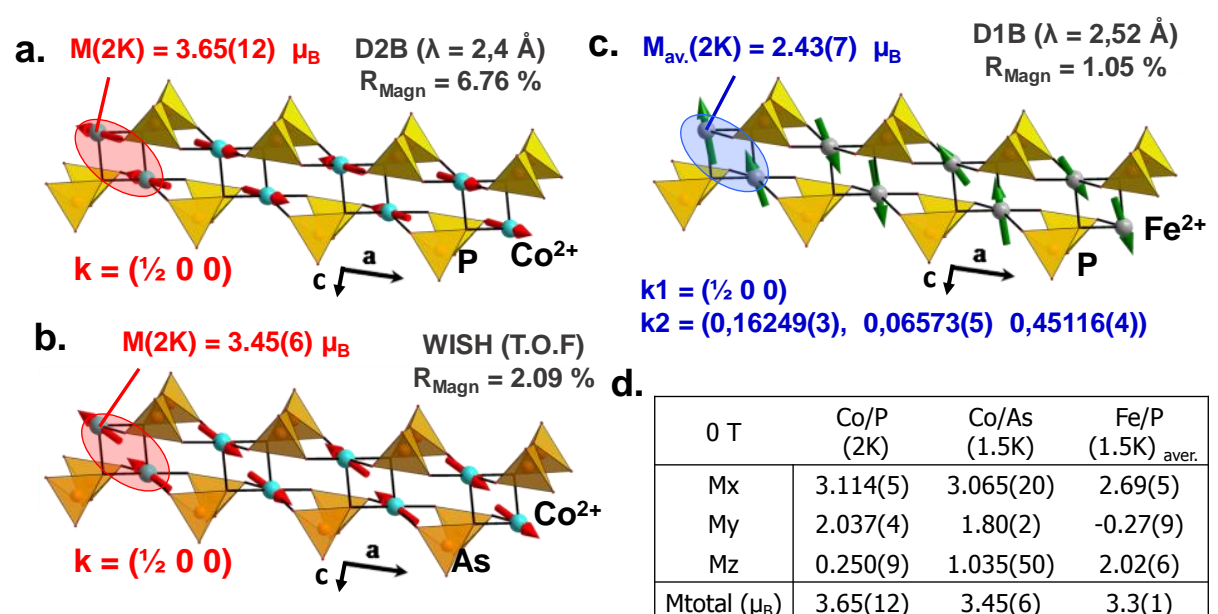

\begin{tabular}{|c|c|c|c|}
\hline $0 \mathrm{~T}$ & $\begin{array}{l}\mathrm{Co} / \mathrm{P} \\
(2 \mathrm{~K})\end{array}$ & $\begin{array}{l}\mathrm{Co} / \mathrm{As} \\
(1.5 \mathrm{~K})\end{array}$ & $\begin{array}{c}\mathrm{Fe} / \mathrm{P} \\
(1.5 \mathrm{~K})_{\text {aver. }}\end{array}$ \\
\hline$M x$ & $3.114(5)$ & $3.065(20)$ & $2.69(5)$ \\
\hline My & $2.037(4)$ & $1.80(2)$ & $-0.27(9)$ \\
\hline $\mathrm{Mz}$ & $0.250(9)$ & $1.035(50)$ & $2.02(6)$ \\
\hline Mtotal $\left(\mu_{\mathrm{B}}\right)$ & $3.65(12)$ & $3.45(6)$ & $3.3(1)$ \\
\hline
\end{tabular}

e.

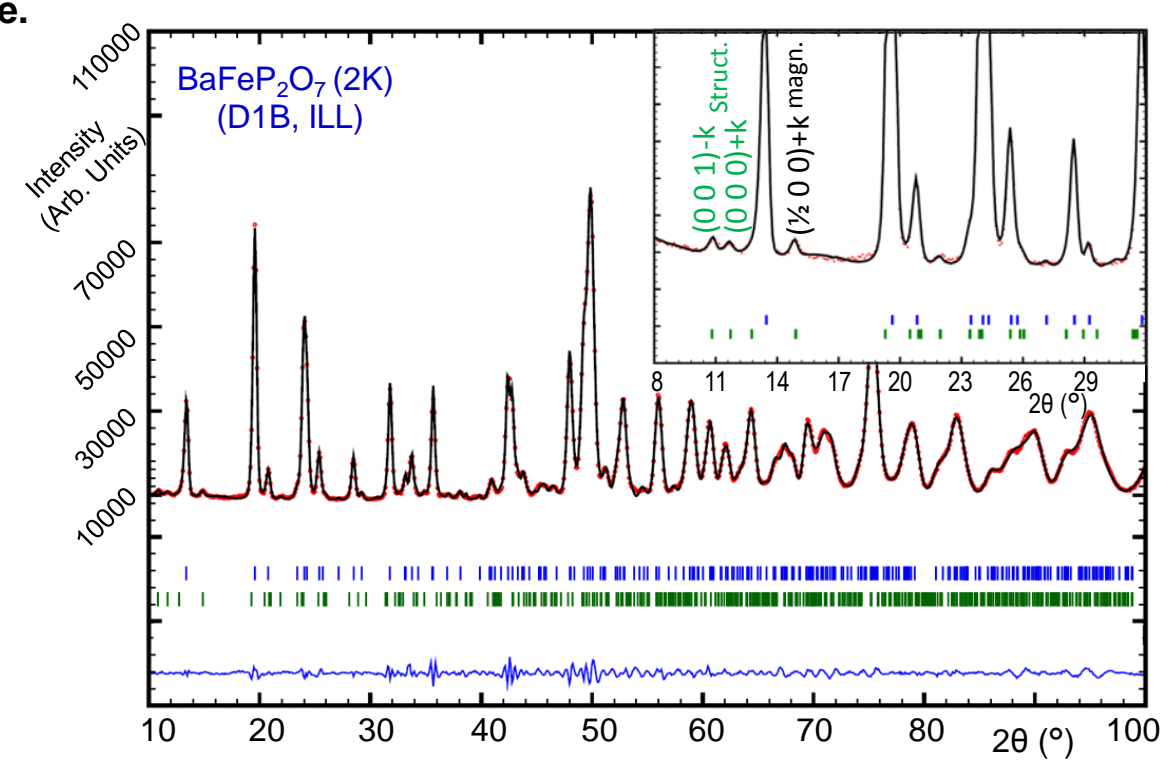

Figure 4 Magnetic Structures: Collinear and modulated spin arrangement for a) $\mathrm{BaCoP}_{2} \mathrm{O}_{7} \mathbf{b}$ ) $\mathrm{BaCoAs}_{2} \mathrm{O}_{7}$ c) $\mathrm{BaFeP}_{2} \mathrm{O}_{7}$ with refined moment values and $\mathrm{k}$ vectors. The FM dimers are highlighted. d) Refined Mx, My, Mz components (full and average for $\mathrm{Co}^{2+}$ and $\mathrm{Fe}^{2+}$ cases respectively). e) Exp., calc. and difference NPD patterns for $\mathrm{BaFeP}_{2} \mathrm{O}_{7}$ (see text for details) with evidences of incommensurate structural (green) and magnetic (black) satellites.

$\mathrm{BaCoAs}_{2} \mathrm{O}_{7}$ Magnetic phase diagram: In addition to its counter-intuitive collinear AFM ground state blind to the displacive atomic wave, the atypical $\mathrm{BaCoAs}_{2} \mathrm{O}_{7} \mathrm{M}(\mathrm{H})$ dependence deserves attention. It was studied by NPD under magnetic field, using the data shown in Figure 3a. We used a densified $\mathrm{BaCoAs}_{2} \mathrm{O}_{7}$ polycrystalline pellet, $a$ priori not sensitive to domains reorientations and tested by collecting data increasing the field from o to $8 \mathrm{~T}$ but also decreasing the field, to verify the recovery of the $1^{\text {st }}$ branch measured intensities. Due to the coexistence of up-to-three magnetically ordred phases, the refinements were performed using the $\mathrm{M}_{(2 \mathrm{~K}, \mathrm{o}-8 \mathrm{~T})}-\mathrm{M}_{(2 \mathrm{oK}, \text { oT) }}$ difference patterns. Under field, only PND data of the $\mathrm{BaCoAs}_{2} \mathrm{O}_{7}$ phase have been exploited due to a high ratio of moisture and high background collected in the phosphate case, see the Supplementary materials So. Dealing with NPD under field, the following quantitative results should be considered with precaution due to the 
formation of purely magnetic textures of orientational character along the magnetic field. In the metamagnetic $\mathrm{BaCoAs}_{2} \mathrm{O}_{7}$, because the field-induced phases appear at the expense of the AFM ground state for specific relative "spin vs. field orientations ", the refinements under field were conducted with respect to : i) the conservation of the sums of the scaling factors with respect to the value refined at o $\mathrm{T}$, in order to probe the same amount of spins at each stage. ii) the use for all phases of "magnetic" preferred orientation along the easy axis, i.e the AFM spin direction [301], in order to model the changing contribution of these domains along with the phase transformations. The Figure $5^{\mathrm{a}}$ displays parts of the difference pattern at $1.5 \mathrm{~K}$ recorded for $\mathrm{H}=0,5.5$ and $8 \mathrm{~T}$. The refined results for these fields values are summarised in Figure $5 e$.

Spin-density-Wave under field: For $\mathrm{BaCoAs}_{2} \mathrm{O}_{7}$ above $\mathrm{H}=2 \mathrm{~T}$, incommensurate magnetic reflections locked on the $\mathrm{k}=\mathrm{q}=[0.13571(4), 0.07806(5), 0.47683(5)$ ] structural q-vector start growing, more intense at high field, see Figure 5a indexed as $\left(\begin{array}{lll}1 & 1 & 0\end{array}\right)-\mathrm{q}(\mathrm{o} \quad 10)-\mathrm{o}$. Here the $\mathrm{Co}^{2+}$ moment of the AFM phase determined at $1.5 \mathrm{~K}$ being very close to the one refined using the full pattern $\left(\mu_{C_{o}}=3.35(8) \mu_{B}\right.$ versus $M=3.45(6)$


contribution during our refinements of the $5.5 \mathrm{~T}$ and $8 \mathrm{~T}$ NPD data. The modulated magnetic structure corresponds to an incommensurate spin density wave (SDW) with the spins almost confined in the $b c$ plane. Our refinement give the variation of the local moments between o and $c a .1 .9 \mu$ nearly perpendicular to their orientation in the Néel ordered state, see Figure 5a,c. This amplitude and direction were fixed equal at 5.5 and $8 \mathrm{~T}$. The resulting refined massic ratios are given in the Figure 5e. Topologically, this SDW is similar to the magnetic structure observed in the quasi-one-dimensional Ising-like $(\mathrm{Ba}, \mathrm{Sr}) \mathrm{Co}_{2} \mathrm{~V}_{2} \mathrm{O}_{8}$ which experimentally also shows a brutal phase transition into a Tomonaga-Luttinger liquid with SDW ${ }^{[12,37]}$.

Ferromagnetic state: Above $7 \mathrm{~T}$, the ferromagnetic contribution appear, mainly observed on the (o 10 ) and (o o 1) reflexions, (see Figure 5d). According to a spin flip scenario, the AFM and FM phases were constrained to have the same moments, without penalisation of the reliability factors. This is also in agreement with the single crystal magnetisation superior along the a and b-axes in agreement with easy magnetic axis mainly along the $a$ direction (see Figure $2 \mathrm{a}$ ).

Although the refined ratio should be considered with much precaution, they picture that the AFM phase is destabilised by the magnetic field and reduces its amount from $100 \%$ (o T) to $74 \%(5.5 \mathrm{~T})$ to $49 \%(8 \mathrm{~T})$, see Figure 5 e. The SDW and FM phases appear at the expense of the AFM one, as shown in Figure 5a. Further assumptions require single crystal data, but a plausible scenario can be proposed, taking into account the easiest softening of the Néel ordering with $\mathrm{H}$ along the AFM spin direction, as confirmed by single crystal magnetization, see Figure $2 \mathrm{a}$. Then, using a densified polycrystalline sample, the evolution of the three phases roughly respects the distribution of the domains towards the external field. The FM state (23\%) at $8 \mathrm{~T}$ is far from the expected amount observed from magnetization which suggests a strong contribution of disordered moments in a forced aligned state and suggest that 
the spins reversal occur preferentially for the grains with spins parallel to $\mathrm{H}$ as expected for a spin flip, while transversal ones preserve the AFM ordering. The SDW then appears as minimal energy option in the intermediate orientations. A synopsis of the behaviour under magnetic field is proposed in the Supplementary Figure 4e.
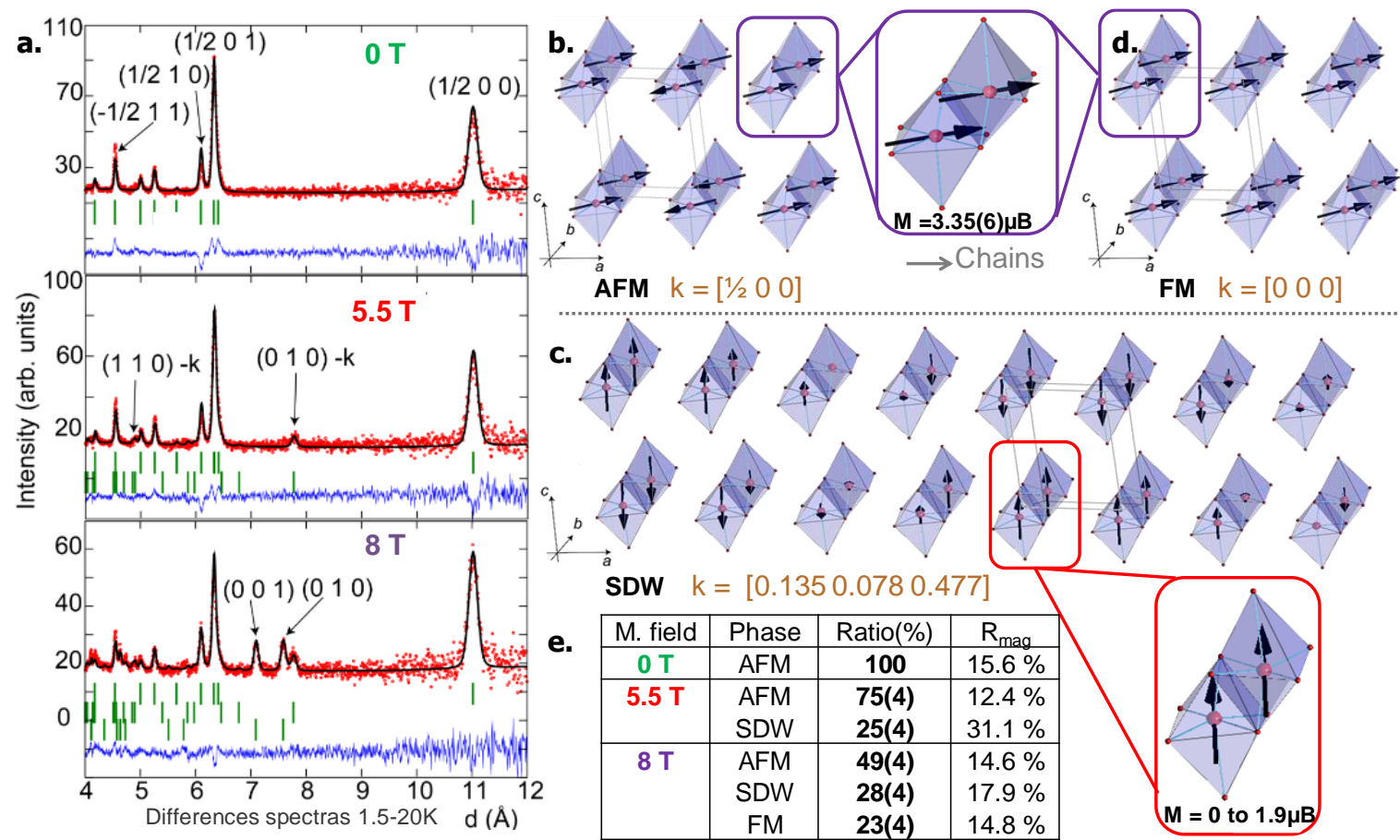

e.

\begin{tabular}{|c|c|c|c|}
\hline M. field & Phase & Ratio(\%) & $R_{\text {mag }}$ \\
\hline 0 T & AFM & $\mathbf{1 0 0}$ & $15.6 \%$ \\
\hline 5.5 T & AFM & $\mathbf{7 5 ( 4 )}$ & $12.4 \%$ \\
& SDW & $\mathbf{2 5 ( 4 )}$ & $31.1 \%$ \\
\hline 8 T & AFM & $\mathbf{4 9 ( 4 )}$ & $14.6 \%$ \\
& SDW & $\mathbf{2 8 ( 4 )}$ & $17.9 \%$ \\
& FM & $\mathbf{2 3 ( 4 )}$ & $14.8 \%$ \\
\hline
\end{tabular}

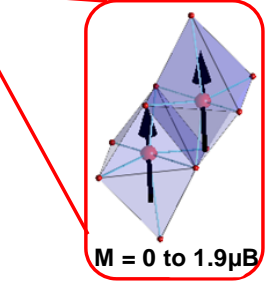

Figure 5 | $\mathrm{BaCoAs}_{2} \mathrm{O}_{7}$ Magnetic structure under magnetic field: a)1.5 $\mathrm{K}-20 \mathrm{~K}$ PND difference patterns at $\mathrm{H}=0,5.5$ and $8 \mathrm{~T}$ and Rietveld refinement profiles using time-of-flight NPD WISH from the bank centred at $58^{\circ}$. Only the AFM phase is present at $\mathrm{H}=\mathrm{o}$ T. AFM and SDW phases coexist at 5.5 T. AFM, SDW and FM phases coexist at $\mathrm{H}=8 \mathrm{~T}$. b-d) Magnetic structures of the variants under field with insets pointing out the FM dimers. e) Resume of the refined fraction and $\mathrm{R}_{\text {magn. }}$ for each phases versus the applied field.

Metamagnetic Transitions of modulated origin: The main magnetic exchanges calculated for the average cells are listed in the Table 1. Considering highly correlated $3 d$ electrons we used GGA+U $\left(\mathrm{U}=4\right.$ for $\mathrm{Co}^{2+}$ and $6 \mathrm{eV}$ for $\left.\mathrm{Fe}^{2+}[18,20,38]\right)$ and the taken magnetic configurations are those preliminary used for $\mathrm{BaCoAs}_{2} \mathrm{O}_{7}{ }^{[21}$, see Supplementary Figure 5 for details (representation of the ordered spin states, values of geometrical parameters along the magnetic exchanges paths, and resulting values of magnetic exchanges parameters). Jdimer are systematically found ferromagnetic correspondingly to the refined magnetic structures. Jchain corresponds to the strongest exchange between two dimers $\left(J_{\text {dimer }} / k_{b}=7.5 \mathrm{~K}\right)$ along the chains and is AFM with sizeable values well correlated to the $\theta_{\mathrm{cw}}$ temperatures. $\mathrm{J}_{\mathrm{c}}$ corresponds to the interchain coupling in the $(a c)$ plane but was estimated neglectable for $\mathrm{BaCoAs}_{2} \mathrm{O}_{7}$ in the average cell $\left(\mathrm{J}_{\mathrm{c}}=-0.8 \mathrm{~K}\right)$. Similarly, no orbital overlap contributes to the exchange along $b\left(\mathrm{~J}_{\mathrm{b}}\right)$, suggesting a very weak value also, which gives a pronounced $1 \mathrm{D}$ topology to the average systems. Although variable intra/inter values between the compounds, the $\mathrm{J}_{\text {dimer }} / \mathrm{J}_{\text {chain }}$ ratio remain close (see Table. 1 , and Supplementary Table 5 for details) leading to similar $\mathrm{T}_{\mathrm{N}} \sim 10 \mathrm{~K}$ in the full series. Here, the undulated atomic strings are necessary tools to bring the frustration into a 
pseudo-2D topology necessary for an abrupt reversal of the spins under magnetic field in $\mathrm{BaCoX}_{2} \mathrm{O}_{7}$ as suggested in our prior work ${ }^{[21]}$. We have used Quantum Monte Carlo (QMC) simulations of $\mathrm{M}(\mathrm{H})$ evolution for $\mathrm{BaCoAs}_{2} \mathrm{O}_{7}$ to validate the modulation role, using both the "average" and a "modulated-approximated" periodic models. The QMC modelization was performed taking Ising $\mathrm{S}=3$ macro-spins to model $\mathrm{Co}^{2+} \mathrm{FM}$ pairs. Details of the calculations are given in Supplementary Figure 5.

For the average model the QMC-calculated $\mathrm{M}(\mathrm{H})$ plot is shown in the Figure $6 \mathrm{c}$, using magnetic exchanges already presented in Table 1 , i.e. $\mathrm{J}_{\text {chain }} / \mathrm{k}_{\mathrm{B}}=-7.5 \mathrm{~K}, \mathrm{~J}_{\mathrm{c}}=-0.8 \mathrm{~K}$. For the 3-D connection, along the b-axis we assumed $\mathrm{J}_{\mathrm{b}}=\mathrm{J}_{\mathrm{c}}$, see Figure 6a. Qualitatively, our quasi-1D "average model" does not reproduce neither the low-field magnetization which experimentally remain unchanged nor the metamagnetic step observed above $5 \mathrm{~T}$ in $\mathrm{BaCoX}_{2} \mathrm{O}_{7}$. However, we calculated a broad flat anomaly at Ms/2 around $6 \mathrm{~T}$ most plausibly assigned to ferrimagnetic metastable states similar to what calculated by QMC for the 1-D $\mathrm{Ca}_{3} \mathrm{Co}_{2} \mathrm{O}_{6}$ compound [39]. Summarizing, it is clear that the average model lacks a pronounced frustrated character to reproduce the abrupt $\mathrm{M}(\mathrm{H})$ step. Indeed, the intensively studied 1/3 magnetization step is theoretically promoted by next-nearest neighbour (NNN) frustration in $1 \mathrm{D}$-isotropic quantum spin chains ${ }^{[40]}$ or by a certain degree of frustration in $2 \mathrm{D}$ triangular lattices ${ }^{41}$.

a. $(a, b, c)$ average cell
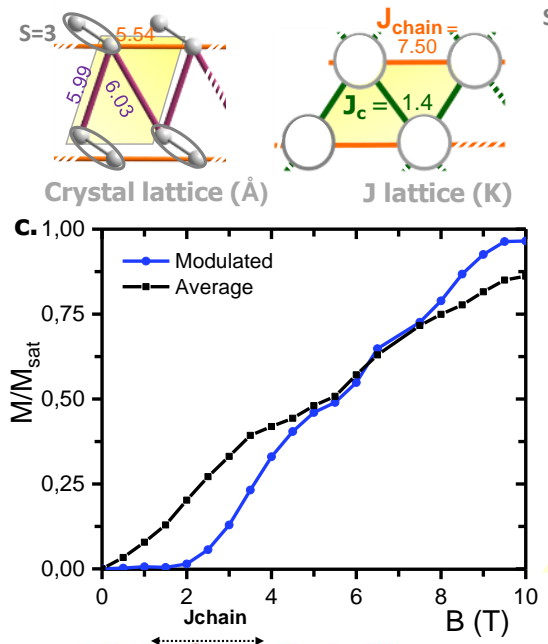

b. $(7 a, b, 2 c)$ Supercell

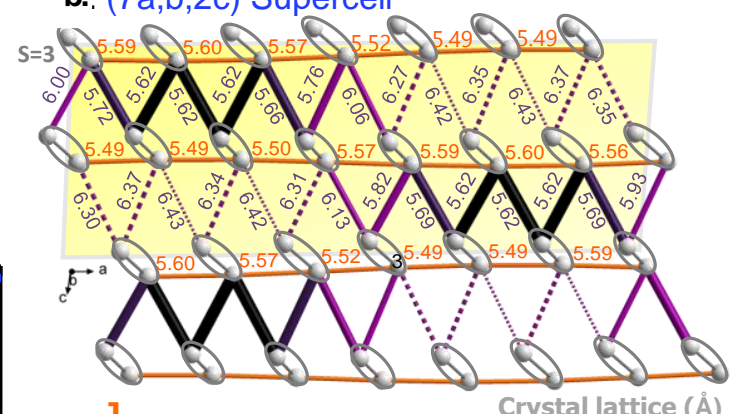

$\mathrm{J}_{\text {chain }}=$ Crystal lattice $(\AA)$

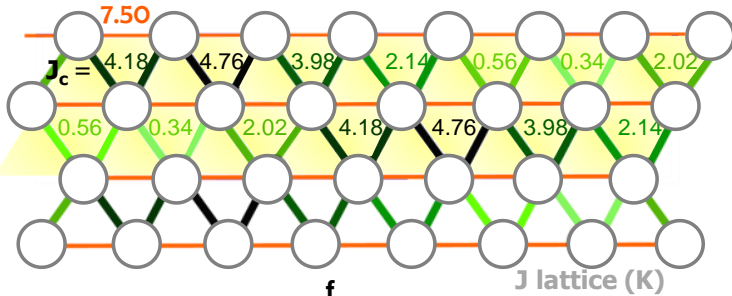

d.
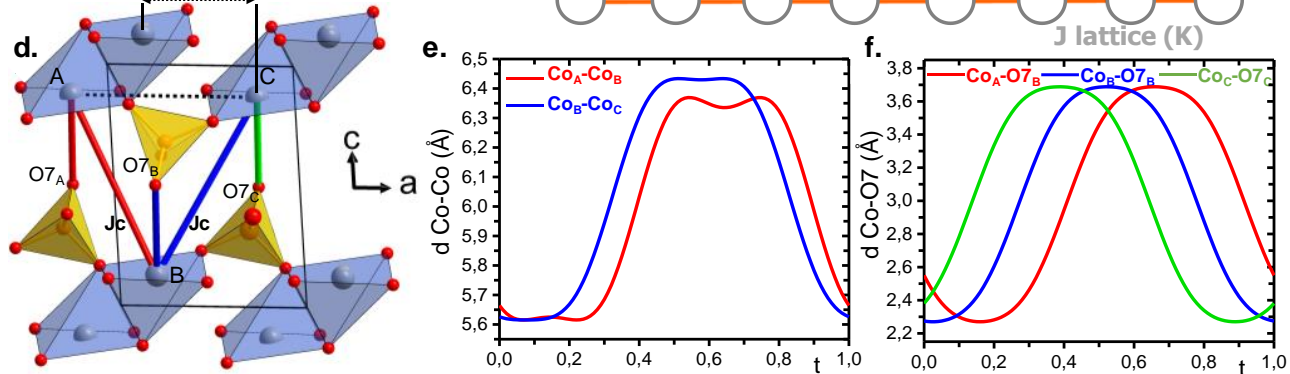

Figure 6 | QMC simulations: a) average crystal structure: atom (left, $d$ in $\AA$ ) and exchange (right, J/kb in $\mathrm{K}$ ) used for the QMC $\mathrm{M}(\mathrm{H})$ simulation using $\mathrm{S}=3$ Ising spins for the FM dimers. The perpendicular $\mathrm{J}_{\mathrm{b}}=-0.8 \mathrm{~K}$ value is weak. b) $7 \mathrm{a}, \mathrm{b}, 2 \mathrm{c}$ approximant for the incommensurate structure with the atom (top) and exchange lattices. Jchain 7.5 and $\mathrm{J}_{b}=-0.8 \mathrm{~K}$ are taken constant. c) QMC M(H) calculations for the two models with the abrupt metamagnetic transition appearing in the pseudo-modulated lattice only. d, e, f) validation of the two equivalent $J_{c}$ values approximation for each $J_{c h a i n}-J_{c}-J_{c}$ triangle plotting the 
involved $\mathrm{Co}-\mathrm{Co}$ and $\mathrm{Co}-\mathrm{O}$ distances which evolve closely in the crystal along the t-modulated real space axis. The bond color of d) are used in e-f) plots.

For the "incommensurate" modelization, the $\mathrm{BaCoAs}_{2} \mathrm{O}_{7}$ modulated lattice with $\mathrm{q}=(0.13571(4), 0.07806(5), 0.47683(5))$ was approximated in a $7 a \times b \times 2 c$ superlattice to approach the real $2 \mathrm{D}$ incommensurate distribution of exchanges, see Figure 6b. Despite severe undulation of the chains along the c-axis the topology along the chain-axis (a-axis) remains approximately constant, which results in a unique J chain exchange of $-7.5 \mathrm{~K}$ for simplification, see Figure 1 . The complex distribution of exchanges in the $(a c)$ plane was estimated using four local structures found in the crystal, at $t=0.15,0.40,0.65,0.90$, see Figure $1 c$, where the real space coordinate was previously defined. They cover the full range of interchain $\mathrm{Co}-\mathrm{Co}$ distances. For each local structure, $\mathrm{J}_{\mathrm{c}}$ was calculated by DFT, plotted against the Co-Co distance and fitted by a linear $\mathrm{J}\left(\mathrm{d}_{\mathrm{Co}-\mathrm{Co}}\right)$ dependence, see Supplementary Figure 5 . Finally, after building the $7 a \times b \times 2 c$ approximant structure each $i^{\text {th }}$ interchain $\mathrm{Co}-\mathrm{Co}$, distance was converted in a specific $\mathrm{J}_{c, i}$ value, leading to the super lattice shown in the Figure $6 \mathrm{~b}$. Here again, we assume two equivalent exchanges per frustrated $\mathrm{J}_{\text {chain, }} \mathrm{J}_{\mathrm{c}}, \mathrm{J}_{\mathrm{c}}$ triangle. The validity of this additional approximation is verified plotting the two $\mathrm{d}(\mathrm{Co}-\mathrm{Co})$ concerned distances that evolve closely in the real space axis, see the Figures $6 \mathrm{~d}, \mathrm{e}, \mathrm{f}$. Also the three $\mathrm{Co}-\mathrm{O}_{7}$ bonds mediating the $\mathrm{Co}_{-} \mathrm{O}_{7}-\mathrm{O}_{7}-\mathrm{Co}$ superexchange paths, show very similar evolutions which comforts the validity of two nearly $\mathrm{J}_{c}$ values in each $(\mathrm{S}=3$ dimer) $)_{3}$ triangle.

The calculated magnetization is shown in the Figure 6c, with evidence of a constant magnetization at low field followed by an abrupt step at $2.5 \mathrm{~T}$ (smaller than the exeperimental $5 \mathrm{~T}$ critical field). This calculated metamagnetic state validates the importance of the frustrated areas in the $(a c)$ plane created by the undulation of the chains. According to our "Ising model" QMC procedure, it represents the setting of spin-flipping that initiates the spin reversal until saturation. Similarly, to our "average" simulation, the QMC anomaly calculated at $\mathrm{M}(\mathrm{H}) / 2$ most possibly represents metastable states offered in the model. At least, although severe approximations, the Figure $6 \mathrm{~b}$ allows visualizing the distribution of $\mathrm{Js}$ in $\mathrm{BaCoX}_{2} \mathrm{O}_{7}$ and questions about the rather simple magnetic structure $(\mathrm{k}=1 / 2 \mathrm{o} \mathrm{o})$ previously reported.

Multiferroicity: Group theory analysis using Isosubgroup [34,35] shows that the experimental GP1 $\mid \mathrm{mX}_{1}+$ irreducible representations is compatible with two magnetic space groups P11'_c(a,b,g)oo and P-11'_c(a,b,g)oo, not distinguishable using NPD due to weak atomic displacements after spin-lattice coupling. The former allows spindriven multiferroicity fulfilling a nonpolar/polar symmetry break, see Supplementary Table. 6.

It was checked measuring the dielectric constant $\varepsilon$ of $\mathrm{BaCoAs}_{2} \mathrm{O}_{7}$ across the AFM transition under applied magnetic fields. Using a single crystal $300 \times 40 \times 20 \mu \mathrm{m}^{3}$ with contacts mounted along the a-axis, i.e. the chain direction, $\varepsilon(T)$ deviates from linearity below $\mathrm{T}_{\mathrm{N}}$ and drastically drops below $7 \mathrm{~K}$. the magnetocapacitance is significant at low temperature (see Figure $7 a)$. The needle-like shape-factor $(e / S)$ of this crystal being problematic for accurate dielectric measurements $(\varepsilon=\mathrm{Cp} \times \mathrm{e} / \mathrm{S})$ we 
show the relative dielectric constant across $\mathrm{T}_{\mathrm{N}}$, defined as $\varepsilon / \varepsilon_{20 \mathrm{~K}}$. A dense pellet was also used, also showing a sensitive divergence of the dielectric constant $\varepsilon(T)$ below $\mathrm{T}_{\mathrm{N}}$ and clear magneto-electric coupling at $9 \mathrm{~T}$, Figure $7 \mathrm{~b}$, see also Supplementary Figure 9. A similar $\varepsilon(\mathrm{T})$ drift was reported for the $\mathrm{CoCr}_{2} \mathrm{O}_{4}$ spinel below $27 \mathrm{~K}$ where the material develops concomitantly a spiral magnetic structure and polarization ${ }^{[42]}$. Polycrystalline pellet and single crystal samples display contrasted deviation from the high temperature interpolation around $\mathrm{T}_{\mathrm{N}}$. However, in the former sample, $\mathrm{dC} / \mathrm{dT}$ precisely locates the anomaly at $\mathrm{T}_{\mathrm{N}}$ proving a weak but clear $\mathrm{ME}$ coupling. Remarkably, the thermal evolution of $\varepsilon$ for polycrystalline $\mathrm{BaFeP}_{2} \mathrm{O}_{7}$ refutes any ME coupling in this compound, see Figure $7 \mathrm{~b}$. It was also checked at $10 \mathrm{~K}$ that no significant magnetodielectric effects occur, see Supplementary Figure 6, which suggests the decisive role of the spin orientation and collinearity in these series.

For $\mathrm{BaCoAs}_{2} \mathrm{O}_{7}$, the magnetodielectric profiles collected below $\mathrm{T}_{\mathrm{N}}$, see Figure 7 c, highlights a sharp increase of $\varepsilon$ even at low magnetic fields, and are symmetric at all temperatures. The magnitude of the magnetodielectric response MD defined as $\left[\varepsilon_{(\mathrm{H})}-\varepsilon_{(\mathrm{H}=\mathrm{o})}\right] / \varepsilon_{(\mathrm{H}=\mathrm{o})}$ increases by a factor of $\sim 2$ from $10 \mathrm{~K}$ (just below $\mathrm{T}_{\mathrm{N}}$ ) to $7 \mathrm{~K}$, and proves ME effects. It reaches $0.03 \%$ at $9 \mathrm{~T}$, similar to what observed in the multiferroic $\mathrm{NiCr}_{2} \mathrm{O}_{4}{ }^{[42,43]}$. It could reasonnably well be fitted using linear $\mathrm{ME}$ couplings, but the $7 \mathrm{~K}$ and $8 \mathrm{~K}$ data also show a sensitive break above $\mathrm{H}=4-5 \mathrm{~T}$, above the critical field for the metamagnetic field. Keeping in mind that at 2 K NPD suggests the appearing of the SDW phase and FM-ordered phase above 2 and $5.5 \mathrm{~T}$ respectively, see the Figure 5, the strictive effect due to forced-alignement of disordred spin component is plausible.

The electric polarization was evaluated by measuring the pyroelectric current on both polycrystalline and single crystal $\mathrm{BaCoAs}_{2} \mathrm{O}_{7}$. Using the pellet, leakage currents were too large for a safe poling. Using cut piece of single crystal with contacts along the [10o] direction, after poling at $30 \mathrm{~K}$ down to $6 \mathrm{~K}$ under $+50 \mathrm{~V}$ a pyrocurrent is developed below $\mathrm{T}_{\mathrm{N}}$. An electrical polarization of $\sim 250 \mu \mathrm{C} / \mathrm{m}^{2}$ is estimated at $6 \mathrm{~K}$ as shown in the Figure $7 \mathrm{~d}$ which comfort the setting of spin-induced polarization out of the centrosymmetric $\mathrm{P}-1(\alpha, \beta, \gamma) \mathrm{O} 4 \mathrm{D}$ super space group at room temperature. The polarization magnitude should be taken carefully due to the approximative geometrical factor used and non homogeneous edge effects of electric field. The anomaly detected below $7 \mathrm{~K}$ is consistent with the $\varepsilon(\mathrm{T})$ drop noted in the Figure $7 \mathrm{a}$, but was not identified magnetically nor structurally at the moment. Ferroelectricity implies the existence of a spontaneous polarization, leading the pyrolectric current, which direction can be fully switched by a sign change of the electric field. Here, the polarization was partially returned after $-50 \mathrm{~V}$ poling, due to inherent difficulties to reverse ferrolectric domains in a single crystalline sample.

All together the co-presence of switchable spin-induced polarization and magneto-electric couplings below $\mathrm{T}_{\mathrm{N}}$ validate the realization of type II multiferroricity, of particular origin. Especially, besides most identified mechanisms for type-II multiferroicity, including the Dzyaloshinsky Moriya inverse (DMI) effect for which a periodic structure with modulated spin structure breaks the space symmetry, $\mathrm{BaCoX}_{2} \mathrm{O}_{7}$ compounds imply a paradoxal situation, where a structurally modulated centrosymmetric lattice together with commensurate collinear spin order develops multiferroicity, see the Figures 
8a,b. On the opposite, magnetoelectric measurements on a $\mathrm{BaFeP}_{2} \mathrm{O}_{7}$ pellet of similar quality, does not show any detectable magnetoelectric effects, see the inset of the Figure $7 a$ and Supplementary Figure 6.

Discussion: Dealing with a collinear spin structure in $\mathrm{BaCoX}_{2} \mathrm{O}_{7}$, the polarization induced between two spins $i$ and $j$ by DMI effect expressed as $\mathrm{P} \propto \mathrm{S}_{i} \mathrm{x} \mathrm{S}_{j}$ is based on SOC but cannot prevail in our compounds, leading to null local terms. The second refutable mechanism is the spin-dependent hybridization model (spin-charge coupling), mediated by the spin-orbit interaction. It is based on the variation of the ionic charge of the oxygen depending on the angle between the spin and the metaloxygen bond, able to generate polarization in absence of atomic shift. According to this mechanism the local polarization of the $\mathrm{CoO}_{5+1}$ polyhedra is expressed by $\mathrm{P} \propto$ $\sum_{i=1}^{5-6}\left(\text { S. }_{\mathrm{i}}\right)^{2} \mathrm{e}_{\mathrm{i}}$ where $\mathrm{e}_{\mathrm{i}}$ is the unit vector along the $\mathrm{i}^{\mathrm{th}} \mathrm{Co}$ - bond. This mechanism, was first reported for $120^{\circ}$ spin structure delafossite [44,45], and also identified in $\mathrm{Ba}_{2} \mathrm{CoGe}_{2} \mathrm{O}_{7}$ with obvious chemical ${ }^{[46]}$ resemblances dealing with the $\mathrm{BaO}-\mathrm{CoO}-\mathrm{X}_{2} \mathrm{O}_{5}$ chemical system. However, in $\mathrm{BaCoAs}_{2} \mathrm{O}_{7}$ the spins are lying in the $\mathrm{O}_{4}$ square plane, while in-plane polarization should be cancelled by opposite bonds for a $\mathrm{CoO}_{4}$ ideal square plane. Although the $\mathrm{Co}^{2+}$ centered polyhedra is significant distorted in plane, its ${ }_{4} \mathrm{D}$-centrosymmetric distribution and elongation with regard to the $\mathrm{P}-1(\alpha, \beta, \gamma)_{0}$ superspace group refutes any significant polarization along the chain axis.
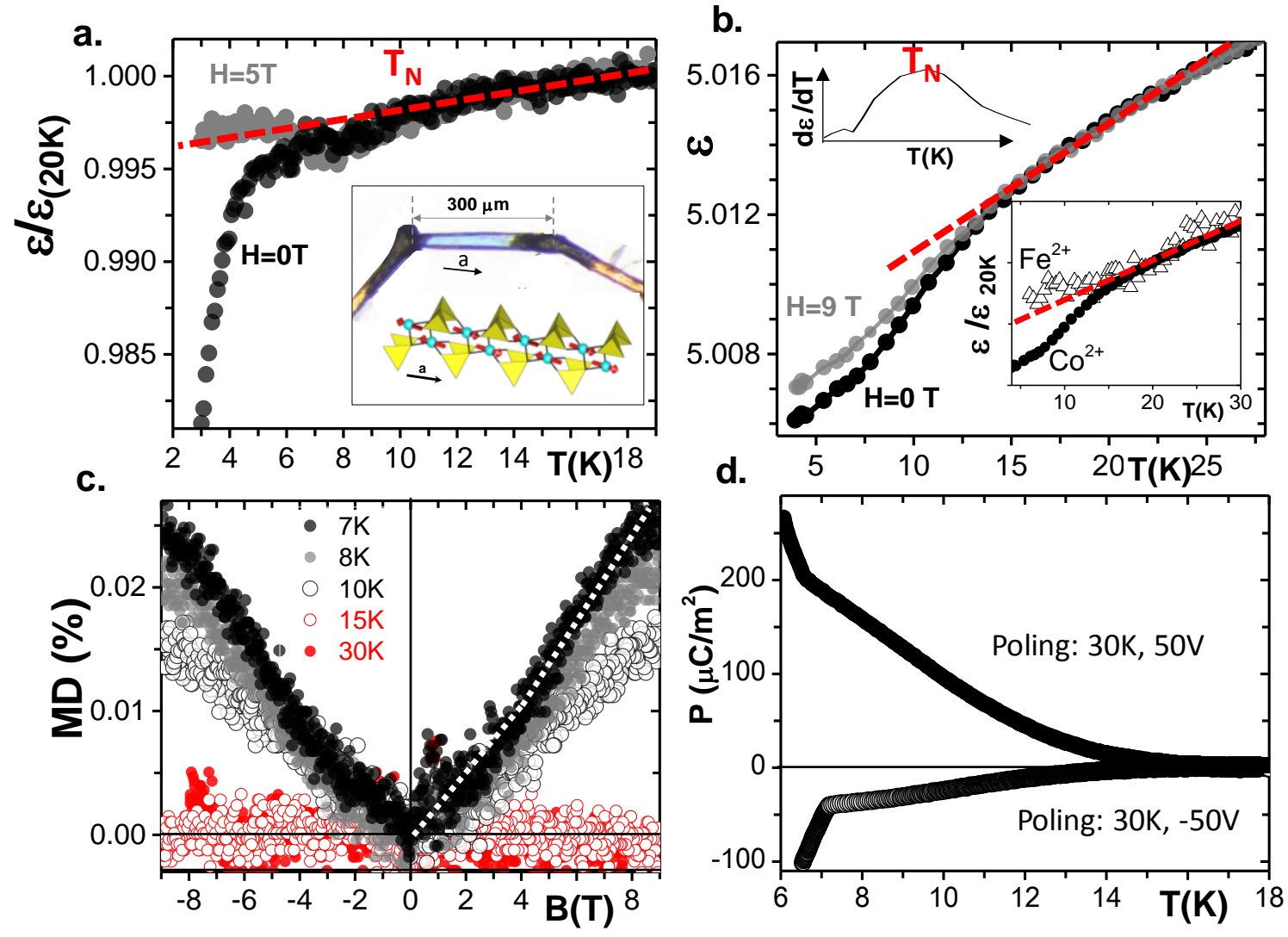

Figure 7 | Magnetoelectric properties a) relative dielectric constant at $\mathrm{H}=\mathrm{o}$ and $5 \mathrm{~T}$ for a $\mathrm{BaCoAs}_{2} \mathrm{O}_{7}$ needle shaped crystal. b) similar measurement at $\mathrm{o}$ and ${ }_{9} \mathrm{~T}$ on dense pellet. The inset shows no thermal dependence of the relative $\varepsilon$ for $\mathrm{BaFeP}_{2} \mathrm{O}_{7}$ c) Magneto dieletric (MD) profiles at various temperature. The white broken line is a guide for the eye. d) Pyroelectric measurement (poling) performed on $\mathrm{BaCoAs}_{2} \mathrm{O}_{7}$ single crystal, with evidence of polarity below $\mathrm{T}_{\mathrm{N}}$. 
Then, the third proposed mechanism concerns the exchange-striction proposed in antiferromagnetic collinear systems, such as the Ising chain $\mathrm{Ca}_{3} \mathrm{CoMnO}_{6}{ }^{[22]}$. This mechanism relies on the loss of spatial symmetry in specific $\uparrow \uparrow \downarrow \downarrow$ magnetic units, scaled by the inner $S_{i} . S_{j}$ scalar product within a direct spin-lattice coupling, blind to the particular directions of magnetic moments. In the AFM chains of $S=3$ dimers, this mechanism is cancelled and not discriminant between the spin-collinear $\mathrm{Co}^{2+}$ but spin-modulated $\mathrm{Fe}^{2+}$ compounds. Finally no clear scenario emerges, complicated by incommensurate structural topology. It is uncertain to assess if the collinear structure found for $\mathrm{Co}^{2+}$ compound is a necessary ingredient for multiferroicity. Indeed it was verified by group theory analysis ${ }^{[34,35]}$ that similarly to the inversionbreaking allowed for $\mathrm{BaCoX}_{2} \mathrm{O}_{7}$ after spin-lattice coupling, it is also allowed for $\mathrm{BaFeP}_{2} \mathrm{O}_{7}$ after coupling the modulated superspace group with the two magnetically active $\mathrm{k}$ vectors $(1 / 2 \mathrm{oo}$ and $\alpha \beta \gamma)$ in the polar subgroup $\mathrm{P} 1(a, b, g)_{0}$.

Therefore searching for contrasted argument for the paraelectric to ferroelectric destabilization for the multiferroic $\mathrm{Co}^{2+}$ materials compared with the ME-inert $\mathrm{Fe}^{2+}$ may rely on the spin orientations via magnetic dipole dipole (MDD) interactions. These nanoscale forces are known to have significant effects self-assembly of magnetic particles ${ }^{[47]}$, but can also play a decisive role in the orientations of spins in bulk-magnetic structures when surpassing antagonist SOC effects, see for instance $\mathrm{Sr}_{3} \mathrm{Fe}_{2} \mathrm{O}_{5}{ }^{[48]}, \mathrm{MAs}_{2} \mathrm{O}_{6}(\mathrm{M}=\mathrm{Mn}, \mathrm{Co}, \mathrm{Ni})^{[49]}, \mathrm{Mn}_{4} \mathrm{Ta}_{2} \mathrm{O}_{9}{ }^{[50]}$. We argued in the $\mathrm{BaMX}_{2} \mathrm{O}_{7}$ series that the sizeable $\mathrm{SOC}$ drives the moments nearly in- $(a b)$ plane $\left(\mathrm{Co}^{2+}\right.$ compounds) versus perpendicular to the chains $\left(\mathrm{Fe}^{2+}\right.$ compound) and one may propose the MDD as destabilizing ingredients in the multiferroic cases. The $\mathrm{m}_{\mathrm{j}}$ to $\mathrm{m}_{\mathrm{k}}$ MDD interaction is given by:

$$
M D D: H=-\left(\frac{\mu_{B}{ }^{2}}{a_{0}{ }^{3}}\right)\left(\frac{a_{0}}{r_{j k}}\right)^{3}\left[3\left(m_{j} \cdot e_{j k}\right)\left(m_{k} \cdot e_{j k}\right)-m_{j} \cdot m_{k}\right]
$$

Where $m_{j, k}$ are refined moments in $\mu_{B}, a_{0}$ is the Bohr radius (o.529 $177 \AA$, $r_{j k}$ is the distance between the spin sites $j$ and $k$, and $e_{j k}$ is the unit vector along the inter-site distance. $\left(\mu_{\mathrm{B}}{ }^{2} / \mathrm{a}_{0}{ }^{3}\right)=0.181 \mathrm{meV}$.

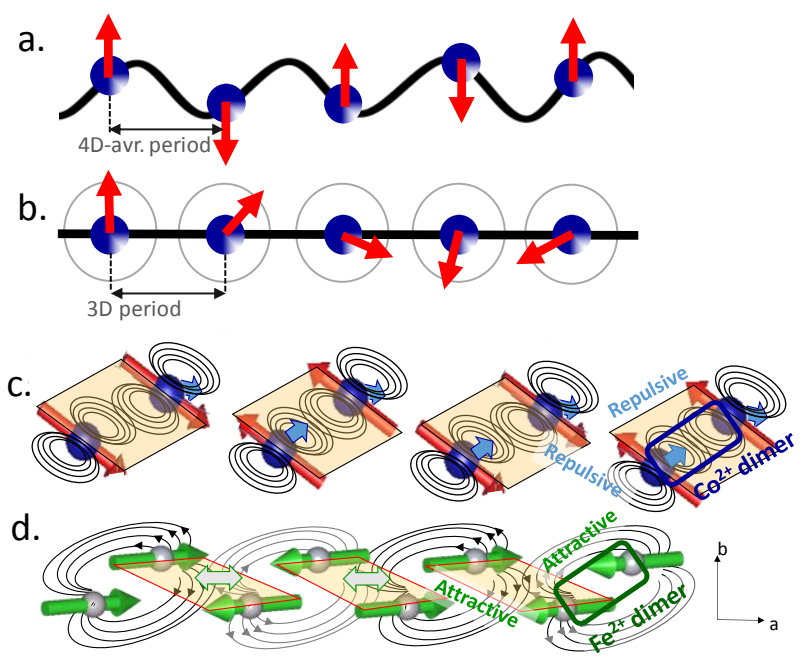


Figure 8 | Proposed ME mechanism: paradox between a) the incommensurate modulated atomic string with collinear spins and $\mathbf{b}$ ) the modulated spin structure over a 3 D lattice (IDM case) $\mathbf{c}$ ) repulsive vs d) attractive dipole-dipole magn. Interaction in $\mathrm{Co}^{2+}$ (with possible atomic shift shown by blue arrows) against $\mathrm{Fe}^{2+}$ compounds

We summed up all of the MDD terms whose distances $\mathrm{r}_{\mathrm{ij}}$ are shorter than a cutoff distance $\mathrm{r}_{\mathrm{c}}=10.5 \AA$ (see Supplementary Tables 7 ) after which contributions were neglectable using the refined average moment vectors in the $\mathrm{Fe}$ case. We found destabilizing (positive) and stabilizing energies for $\mathrm{BaCoX}_{2} \mathrm{O}_{7}\left(+14.4810^{-3} \mathrm{meV}\right.$ for As and $+23.7610^{-3} \mathrm{meV}$ for $\mathrm{P}$, per Co site) and $\mathrm{BaFeP}_{2} \mathrm{O}_{7}\left(-2.8810^{-3} \mathrm{meV}\right.$ per Fe site) respectively and MDD could be a driving force to develop the experimental spininduced polarity. In addition the intra-dimer dipolar interactions (Jdd dimer) and interdimer between two next metal sites ( $\mathrm{Jdd}_{\mathrm{dd}}$ chain) give both repulsive interactions in $\mathrm{BaCoX}_{2} \mathrm{O}_{7}$ compounds against both attractive ones in $\mathrm{BaFeP}_{2} \mathrm{O}_{7}$ due to the spin orientations, see the Figures $8 \mathrm{c}$,d. The calculated $\mathrm{J}_{\mathrm{dd}}$ interactions are given Table 1 and detailled in Supplementary Tables 7 for all counted neighbors. Athough weaker, one notes the same order of magnitude of Jdd's compared to the through-bond J exchanges which denote significant through-space effects.

While the destabilization into a polar mode of the crystal structure by MDD couplings appear vain in a regular lattice due to next-neighbor cancellation, dipoledipole ordering was recently proposed to explain magnetolectric anomaly at the setting of low-D ordering in 1-D materials ${ }^{[5]}$. Practically, dealing with aperiodic materials, infinite periodicities within structural chains, versatile $\mathrm{Co}^{2+}$ local coordinations along them, and their slicing by local defects, the destabilization by cooperative repulsive striction effects may be proposed to generate a polar mode. Even if cooperative, atomic displacements in one chain are not expected regular and should be shifted between chains due to the $3 \mathrm{D}$-structural modulation giving to each Co-site local specificities. Therefore, one can imagine a polar mode mechanism relaxing the MDD stress from chain-to chain.In opposite the energetic stabilization of $\mathrm{BaFeP}_{2} \mathrm{O}_{7}$ by MDD would preserve the spatial inversion from site to site, in good agreement with the attractive interactions along the chains which cancel from site to site.

Besides the proposition of a novel type II multiferroic series of materials, the possibility for an original mechanism for spin-induced polorization by mixing several ingredients such as the SOC influence into uniaxial spin structure, incommensurate atomic strings and well adapted dipole-dipole interaction offers a new paradigm in this field. The aperiodic lattice contribution to magneto-electric exchanges was already proposed in $\mathrm{Cr}$-doped multiferroic $\mathrm{BiFeO}_{3}{ }^{52}$, but more generally our results open wide perspectives about the screening of incommensurate modulated materials so far not considered as multiferroics due to the identified conventional mechanisms.

\section{ASSOCIATED CONTENT}

Supporting Information guidelines 
The supporting information is available free of charge on the .... Website at DOI:

The files (PDF) gives detailed information related to the structural and single crystal refinement for $\mathrm{BaFeP}_{2} \mathrm{O}_{7}$, additional specific heat plots, extra-crystallographic details and magnetic plots for $\mathrm{Fe} / \mathrm{Co}$ mixed compounds, details related to magnetic structures and NPD refinement (such as refinement of $\mathrm{BaFeP}_{2} \mathrm{O}_{7}$ structure in a nonmodulated magnetic case), details for DFT calculations, magnetoelectrics couplings, magnetic dipole-dipole calculations, SEM-EDS analysis, spectroscopies measurements, and lastly additional information related to TGA measurement and proofs of significant moisture in "non-fresh" cobalt compounds. This file contains 26 pages, 9 parts, 14 figures and 16 Tables.

One can find X-Ray crystallographic data for $\mathrm{BaFeP}_{2} \mathrm{O}_{7}$ average + modulated structures CSD 1996565 and the related Check-cif report (PDF).

\section{AUTHORS INFORMATION}

Corresponding Author.

*olivier.mentre@univ-lille.fr

\section{ORCID}

Bastien Leclercq : 0000-0002-3165-322237

Angel M. Arevalo-Lopez : $0000-0002-8745-4990$

Houria Kabbour : oooo-0002-9081-3261

Claire V. Colin : oooo-0003-1332-7929

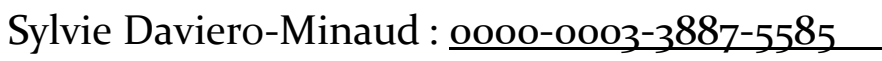

Alain Pautrat:

Tathamay Basu : oooo-0002-8486-189X

Ranjana-Rani Das : 0000-0002-9781-3432

Vaclav Petricek: $0000-0002-9731-8730$

Rénald David :

Olivier Mentré : $\underline{0000-0002-1822-6003}$

\section{Notes}

The authors declare no competing financial interest.

\section{ACKNOWLEDGMENT}

This work was carried out under the framework of the LOVE-ME project supported by the ANR (Grant ANR ANR-16-CEo8-0023). AMAL thanks a Marie-Słodowska Individual Fellowship in the Horizon 2020 research and innovation programme under grant agreement 750971 ("KISS ME”). The Fonds Européen de Développement Régional (FEDER), CNRS, Région Hauts-de-France and Ministère de l'Education 
Nationale, de l'Enseignement Supérieur et de la Recherche are acknowledged for funding of the PPMS and the X-ray diffractometers.. Chevreul Institute (FR 2638), Ministère de l'Education Nationale, de l'Enseignement Supérieur et de la Recherche, Région Hauts-de-France, FEDER are acknowledged for supporting this work. We also thanks the GDR MEETICC for the funding through the "Soutien aux jeunes chercheurs » supply. We thank Laurence Burylo, Nora Djellal \& Claire Minaud at the UCCS for their experimental contribution. We also acknowledge the CRG- DiB, Institut Laue-Langevin at Grenoble, France for beam time allocation and the technical assistance of V. Nassif, S. Djellit. BL, OM and AAL acknowledge Vaclav petricek for his advice refining concomitantely modulated crystal and magnetic structures in absence of magnetic fields.

\section{REFERENCES}

[1] T. Hu, E. Kan, Wiley Interdiscip. Rev. Comput. Mol. Sci. 2019, 9, 1.

[2] S. Dong, J.-M. M. Liu, S.-W. W. Cheong, Z. Ren, Adv. Phys. 2015, 64, 519.

[3] N. A. Hill, J. Phys. Chem. B 2000, 104, 6694.

[4] R. Ramesh, S. N. A., Nat. Mater. 2007, 6, 21.

[5] J. F. Scott, Phys. Rev. B 1977, 16, 2329.

[6] M. Fiebig, T. Lottermoser, D. Meier, M. Trassin, Nat. Rev. Mater. 2016, $1,1$.

[7] S. Dong, H. Xiang, E. Dagotto, Natl. Sci. Rev. 2019, 6, 629.

[8] W. Eerenstein, N. D. Mathur, J. F. Scott, Nature 2006, 442, 759.

[9] M. Lenertz, A. Dinia, S. Colis, O. Mentré, G. André, F. Porcher, E. Suard, J. Phys. Chem. C 2014, 118, 13981.

[10] K. Singh, A. Maignan, D. Pelloquin, O. Perez, C. Simon, J. Mater. Chem. 2012, 22, 6436.

[11] T. Giamarchi, C. Rüegg, O. Tchernyshyov, Nat. Phys. 2008, 4, 198.

[12] E. Canévet, B. Grenier, M. Klanjšek, C. Berthier, M. Horvatić, V. Simonet, P. Lejay, Phys. Rev. B 2013, 87, 054408.

[13] B. Leclercq, H. Kabbour, F. Damay, C. V. Colin, A. Pautrat, A. M. Arevalo-Lopez, O. Mentré, Inorg. Chem. 2019, 58, 12609.

[14] R. David, H. Kabbour, S. Colis, O. Mentré, Inorg. Chem. 2013, 52, 13742.

[15] L. Bogani, A. Vindigni, R. Sessoli, D. Gatteschi, J. Mater. Chem. 2008, 18, 4750.

[16] D. Gatteschi, A. Vindigni, in (Eds.: J. Bartolomé, F. Luis, J.F. Fernández), Springer Berlin Heidelberg, Berlin, Heidelberg, 2014, pp. 191-220.

[17] B. Leclercq, H. Kabbour, A. Arevalo-Lopez, M. Huvé, S. Daviero-Minaud, C. Minaud, I. Blazquez Alcover, O. Mentré, Inorg. Chem. Front. 2019, 7, 239. 
[18] R. David, A. Pautrat, D. Filimonov, H. Kabbour, H. Vezin, M.-H. Whangbo, O. Mentré, J. Am. Chem. Soc. 2013, 135, 13023.

[19] L. P. Regnault, P. Burlet, J. Rossat-Mignod, Phys. B+C 1977, 86-88, 660.

[20] H. Kabbour, R. David, A. Pautrat, H. Koo, M. Whangbo, G. André, O. Mentré, Angew. Chemie Int. Ed. 2012, 51, 11745.

[21] R. David, H. Kabbour, S. Colis, A. Pautrat, E. Suard, O. Mentré, J. Phys. Chem. C 2013, 117, 18190 .

[22] Y. J. Choi, H. T. Yi, S. Lee, Q. Huang, V. Kiryukhin, S. W. Cheong, Phys. Rev. Lett. 2008, 100,6 .

[23] S. Petit, Physics (College. Park. Md). 2013, 6, 4.

[24] A. A. Belik, M. Azuma, M. Takano, Inorg. Chem. 2005, 44, 7523.

[25] D. Riou, H. Leligny, C. Pham, P. Labbe, B. Raveau, Acta Crystallogr. Sect. B 1991, 47,608 .

[26] A. Moquine, etudes chimique, structural et magnetique de nouveaux mono- et diphosphate apparaissant dans les diagrammes ternaires AO-CuO-P2O5; A $=\mathrm{Mg}, \mathrm{Ca}, \mathrm{Sr}$ , PhD manuscript, 1990.

[27] L. Cario, A. Meerschaut, B. Corraze, O. Chauvet, Mater. Res. Bull. 2005, 40, 125.

[28] V. M. Kovrugin, E. E. Gordon, E. E. Kasapbasi, M. H. Whangbo, M. Colmont, O. I. Siidra, S. Colis, S. V. Krivovichev, O. Mentré, J. Phys. Chem. C 2016, 120, 1650.

[29] J. Olchowka, M. Colmont, A. Aliev, T. T. Tran, P. S. Halasyamani, H. Hagemann, O. Mentré, CrystEngComm 2017, 19, 936.

[30] Y. Tsujimoto, Y. Baba, N. Oba, H. Kageyama, T. Fukui, Y. Narumi, K. Kindo, T. Saito, M. Takano, Y. Ajiro, K. Yoshimura, J. Phys. Soc. Japan 2007, 76, 3.

[31] S. M. Yusuf, A. K. Bera, C. Ritter, Y. Tsujimoto, Y. Ajiro, H. Kageyama, J. P. Attfield, Phys. Rev. B - Condens. Matter Mater. Phys. 2011, 84, 1.

[32] Z. He, T. Taniyama, T. Kyômen, M. Itoh, Phys. Rev. B 2005, 72, 172403.

[33] Y. Kawasaki, J. L. Gavilano, L. Keller, J. Schefer, N. B. Christensen, A. Amato, T. Ohno, Y. Kishimoto, Z. He, Y. Ueda, M. Itoh, Phys. Rev. B 2011, 83, 064421.

[34] B. J. Campbell, H. T. Stokes, D. E. Tanner, D. M. Hatch, J. Appl. Crystallogr. 2006, $39,607$.

[35] H. T. Stokes, D. M. Hatch, B. J. Campbell, n.d.

[36] R. R. Das, C. V. Colin, Investigating Antiferromagnetic Magnetic Ground State of a Modulated Phase with Metamagnetic Properties of $\mathrm{BaFeP}_{2} \mathrm{O}_{7}$, Institut Laue-Langevin (ILL),

[37] A. Okutani, T. Kida, T. Usui, T. Kimura, K. Okunishi, M. Hagiwara, Phys. Procedia 2015, 75, 779 . 
[38] A. L. Dalverny, J. S. Filhol, F. Lemoigno, M. L. Doublet, J. Phys. Chem. C 2010, $114,21750$.

[39] R. Soto, G. Martínez, M. N. Baibich, J. M. Florez, P. Vargas, Phys. Rev. B 2009, 79, 184422.

[40] A. Honecker, J. Schulenburg, J. Richter, J. Phys. Condens. Matter 2004, 16, S749.

[41] H. Nishimori, S. Miyashita, J. Phys. Soc. Japan 1986, 55, 4448.

[42] N. Mufti, A. A. Nugroho, G. R. Blake, T. T. M. Palstra, J. Phys. Condens. Matter 2010, 22, 075902.

[43] T. D. Sparks, M. C. Kemei, P. T. Barton, R. Seshadri, E. D. Mun, V. S. Zapf, Phys. Rev. B - Condens. Matter Mater. Phys. 2014, 89, 2.

[44] T. Kimura, Annu. Rev. Condens. Matter Phys. 2012, 3, 93.

[45] S.-W. Cheong, M. Mostovoy, Nat. Mater. 2007, 6, 13.

[46] H. Murakawa, Y. Onose, S. Miyahara, N. Furukawa, Y. Tokura, Phys. Rev. Lett. 2010, 105,1 .

[47] K. J. M. Bishop, C. E. Wilmer, S. Soh, B. A. Grzybowski, Small 2009, 5, 1600.

[48] H. J. Koo, H. Xiang, C. Lee, M. H. Whangbo, Inorg. Chem. 2009, 48, 9051.

[49] H. J. Koo, M. H. Whangbo, Inorg. Chem. 2014, 53, 3812.

[50] N. Narayanan, A. Senyshyn, D. Mikhailova, T. Faske, T. Lu, Z. Liu, B. Weise, H. Ehrenberg, R. A. Mole, W. D. Hutchison, H. Fuess, G. J. McIntyre, Y. Liu, D. Yu, Phys. Rev. B 2018, 94, 1 .

[51] T. Basu, C. Bloyet, J. M. Rueff, V. Caignaert, A. Pautrat, B. Raveau, G. Rogez, P. A. Jaffrès, J. Mater. Chem. C 2018, 6, 10207.

[52] X. Tang, M. J. Gentiletti, A. Lachgar, V. A. Morozov, B. I. Lazoryak, Solid State Sci. 2001, 3, 143. 


\section{Table of Content}

$\mathrm{BaCoX}_{2} \mathrm{O}_{7}(\mathrm{X}=\mathrm{P}, \mathrm{As})$ are shown to be a type II multiferroics based on an original scenario in which incommensurate atomic shifts and collinear AFM ising-spins interact as crucial ingredients. In comparison no magneto-electric coupling was detected in the Heisenberg $\mathrm{BaFeP}_{2} \mathrm{O}_{7}$ isomorph, with strong spin-lattice coupling leading to a modulated AFM spin structure. Magnetic dipole-dipole (MDD) interactions are discreminating between the two systems.

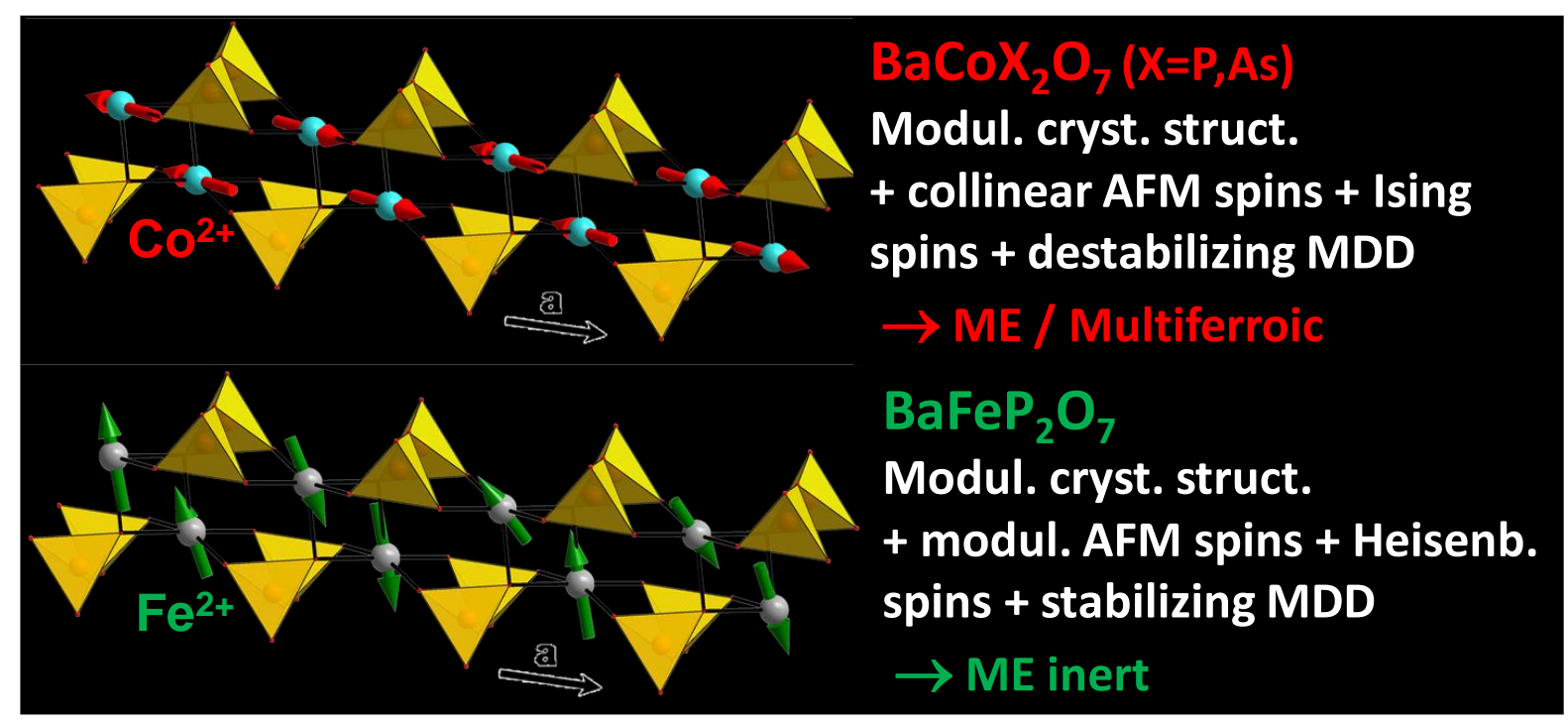

